\title{
Physics and Astrophysics of Strange Quark Matter
}

\author{
Jes Madsen \\ Institute of Physics and Astronomy, University of Aarhus \\ DK-8000 Arhus C, Denmark
}

August 15, 2018

\begin{abstract}
3-flavor quark matter (strange quark matter; SQM) can be stable or metastable for a wide range of strong interaction parameters. If so, SQM can play an important role in cosmology, neutron stars, cosmic ray physics, and relativistic heavy-ion collisions. As an example of the intimate connections between astrophysics and heavy-ion collision physics, this Chapter gives an overview of the physical properties of SQM in bulk and of small-baryon number strangelets; discusses the possible formation, destruction, and implications of lumps of SQM (quark nuggets) in the early Universe; and describes the structure and signature of strange stars, as well as the formation and detection of strangelets in cosmic rays. It is concluded, that astrophysical and laboratory searches are complementary in many respects, and that both should be pursued to test the intriguing possibility of a strange ground state for hadronic matter, and (more generally) to improve our knowledge of the strong interactions.
\end{abstract}

\section{Introduction}

Hadronic matter is expected to undergo a transition to quark-gluon plasma under conditions of high temperature and/or baryon chemical potential. These conditions may be achieved for a brief moment in ultrarelativistic heavy-ion collisions, but they are also likely to appear in Nature. A very high density (and comparatively low temperature) environment exists in the interior of neutron stars, which may actually contain significant amounts of quark matter in the interior. High temperatures (but rather low baryon chemical potential) were realized in the first $10^{-4}$ seconds after the Big Bang, and here a hot quark-gluon plasma state must have existed until the temperature dropped to 100-200 MeV due to the adiabatic expansion of the Universe.

This Chapter will outline some of the possible ways in which astrophysics may teach us about the existence and properties of quark-gluon plasmas. The advantage 
relative to laboratory searches is, that truly bulk systems can be studied, and that the timescales involved are much longer than those relevant to collisions. Disadvantages are that astrophysicists (with possible exceptions if strange quark matter is absolutely stable) can only observe indirect consequences of the plasma state for example in the properties of pulsars or in the distribution of light nuclei produced a few minutes after the Big Bang. It will be shown, however, that astrophysics arguments in many cases can be used to constrain parameters significantly relative to direct experimental approaches because of the large volumes and timescales involved.

The implications of quark-gluon plasmas in astrophysics and cosmology are manyfold, and I shall focus on aspects related to the idea of (meta)stability of strange quark matter through discussions partly biased by my own research interests.

Lumps of up, down, and strange quarks (strange quark matter, SQM), with masses ranging from small nuclei to neutron stars, rather than ${ }^{56} \mathrm{Fe}$, could be the ground state of hadronic matter even at zero temperature and pressure. This possibility, first noted by Bodmer in 1971 [1], has attracted much attention since Witten resurrected the idea in 1984 [2]. The existence of stable or metastable SQM would have numerous consequences for physics and astrophysics, and testing some of these consequences should ultimately tell us whether SQM really exists.

First it was believed that SQM might give a natural explanation of the cosmological dark matter problem. While not ruled out, this idea is now less popular, but strange quark matter may still be important in astrophysical settings, such as strange stars. Numerous investigations have searched for deposits of SQM on the Earth and in meteorites, so far unsuccessfully, and recently relativistic heavy-ion collision experiments have been performed and/or proposed to test the idea. Cosmic ray searches have come up with a few potential candidates for small SQM-lumps (strangelets), but at present no compelling evidence for stable SQM has been presented. This, however, does not rule it out. Most searches for SQM are sensitive to strangelets with very low baryon number, $A$, and as discussed later, finite size effects have a significant destabilizing effect on such objects, even if SQM is stable in bulk.

There is a significant range of strong interaction parameters for which SQM in bulk is stable. But even if it is not, many of the (astro)physical implications are more or less unchanged in the case of metastable SQM. In neutron stars, for instance, the high pressure brings SQM closer to stability relative to hadronic matter, and it is quite likely, that neutron stars contain cores of strange quark matter, even if SQM is unstable at zero pressure. In relativistic heavy-ion collision experiments, strangelets need "only" survive for $10^{-8}$ seconds to be of interest. In fact, (meta)stable strangelets may be one of the "cleanest" signatures for formation of a quark-gluon plasma in such collisions.

The present review tries to give an account of the status of strange quark matter physics and astrophysics, as of early 1998, but of course not all aspects are covered in equal detail. In particular, nothing is said about the heroic experimental efforts 
to produce strangelets in heavy-ion collisions. A collection of papers describing all aspects of SQM and a list of references to the field through mid-1991 can be found in [3]. An earlier review was given in [4]. Recent reviews include [5, 6, 0, 8, 9, 10, 11, 12], and the thorough reader will notice, that some parts of the present Chapter borrows from my own papers among these since the physics discussed therein remains more or less unchanged. Refs. [11, 12 also discuss the related issue of lumps of metastable strange hadronic matter, which will not be dealt with here.

Section 2 discusses the physics of SQM, starting out with simple estimates of why 3 -flavor quark-matter is likely to be more bound than the 2-flavor alternative, proceeding with more detailed descriptions of SQM in bulk. Smaller systems (strangelets), for which finite-size effects are crucial, are described in Section 3. Most of the results are based on the MIT bag-model, but it is worth stressing from the outset that this should only be viewed as a crude approximation to reality, ultimately to be surpassed by direct QCD-calculations.

Section 4 deals with the possible production of lumps of SQM (often called quark nuggets) in the cosmological quark-hadron phase transition, and the struggle of quark nuggets to survive evaporation and boiling in a hostile environment. It turns out, that only large nuggets are likely to survive, but the physics involved in the destruction process is illuminating as it resembles the (time-reversed) physics involved in strangelet production in heavy-ion collisions. Implications of surviving quark nuggets for Big Bang nucleosynthesis and the dark matter problem are also discussed.

Perhaps the most likely place to discover SQM (even if it is not absolutely stable) is in neutron stars. These could be "hybrid", "strange", or even "mixed" (the first term conventionally used for neutron stars with quark cores; the second for "true" quark stars in case of SQM stability, and the latter for objects with mixed phases of quark matter and nuclear matter). Section 5 describes these stars, their implications for our understanding of pulsars, and the possible connection to the energetic gammaray bursters.

Strangelets surviving from the early Universe or released from strange stars in binary systems have been searched for in cosmic ray detectors and in meteorites and mineral deposits. So far there are only a few potential candidates, but more sensitive experiments will soon be carried out. Section 6 discusses some of the limits obtained. It also presents an astrophysical argument which either improves the Earth-based flux-limits by many orders of magnitude (almost excluding absolutely stable SQM), or predicts that all neutron stars are strange stars, if SQM is stable (the prediction to choose depends on whether any pulsars can be proven to be ordinary neutron stars).

Conclusions and a brief outlook are provided in Section 0 .

\section{Physics of SQM in bulk}




\subsection{Does strange matter conflict with experience?}

At first sight, the possibility that quark matter could be absolutely stable seems to contradict daily life experiences (and experiments) showing that nuclei consist of neutrons and protons, rather than a soup of quarks. If a lower energy state exists, then why are we here? Why have we not decayed into strange quark matter?

The answer to this obvious question is, that (meta)stability of strange quark matter requires a significant fraction of strange quarks to be present. Conversion of an iron nucleus into an $A=56$ strangelet thus demands a very high order weak interaction to change dozens of $u$ - and $d$-quarks into $s$-quarks at the same time. Such a process has negligible probability of happening. For lower $A$ the conversion requires a lower order weak interaction, but as demonstrated later, finite-size effects destabilize small strangelets so that they become unstable or only weakly metastable even if strange quark matter is stable in bulk.

Therefore (meta)stability of strange quark matter does not conflict with the existence of ordinary nuclei. On the other hand, the existence of ordinary nuclei shows, that quark matter composed of $u$ - and $d$-quarks alone is unstable, a fact that will be used later on to place constraints on model parameters.

Another constraint from our mere existence can be placed on the electrical charge of strangelets. If energy is gained by converting ordinary matter into strange quark matter, strangelets with negative quark charge, even if globally neutral due to a cloud of positrons, would have devastating consequences, eating up the nuclei they would encounter. Even a small stable component in the cosmos would be intolerable (but they could still appear as metastable products in heavy-ion collisions, like the recent charge -1 , mass $7.4 \mathrm{GeV}$ event in NA52 at CERN [13]). A positive charge on the quark surface (neutralized by surrounding electrons) is less problematic, because ordinary nuclei will be electrostatically repelled. The barrier has to be of a certain height, though, in order not to impact stellar evolution (see below). Note that neutrons are easily absorbed. As demonstrated later, this has important consequences for quark star formation and can be used to constrain strange matter properties using several astrophysical lines of reasoning. It may even lead to practical applications in energy production, etc. [14].

\subsection{Simple arguments for (meta)stability}

As argued above, quark matter composed of $u$ and $d$-quarks is expected to be unstable (except from 3-quark baryons). Introducing a third flavor makes it possible to reduce the energy relative to a two-flavor system, because an extra Fermi-well is available. The introduction of an extra fermion-flavor makes it possible to increase the spatial concentration of quarks, thereby reducing the total energy. A penalty is paid because the mass of the $s$-quark is high compared to that of $u$ and $d$, so stability is most likely for low $s$-quark mass. 
To make the argument slightly more quantitative, consider non-interacting, massless quarks inside a confining bag at temperature $T=0$, without external pressure. For a massless quark-flavor, $i$, the Fermi momentum, $p_{F i}$, equals the chemical potential, $\mu_{i}$ (throughout the chapter, unless otherwise noted, $\hbar=c=k_{B}=1$; for an introduction to Fermi-gas thermodynamics, see for instance Ref. [15]). Thus the number densities are $n_{i}=\mu_{i}^{3} / \pi^{2}$, the energy densities $\epsilon_{i}=3 \mu_{i}^{4} /\left(4 \pi^{2}\right)$, and the pressures $P_{i}=\mu_{i}^{4} /\left(4 \pi^{2}\right)$. The sum of the quark pressures is balanced by the confining bag pressure, $B$; $\sum_{i} P_{i}=B$; the total energy density is $\epsilon=\sum_{i} \epsilon_{i}+B=3 \sum_{i} P_{i}+B=4 B$, and the density of baryon number is $n_{B}=\sum_{i} n_{i} / 3$. Notice that the sum of the constituents pressures, as well as the total energy density are given solely in terms of the bag constant, $B$.

For a gas of $u$ and $d$-quarks charge neutrality requires $n_{d}=2 n_{u}$, or $\mu_{2} \equiv$ $\mu_{u}=2^{-1 / 3} \mu_{d}$. The corresponding two-flavor quark pressure is $P_{2}=P_{u}+P_{d}=$ $\left(1+2^{4 / 3}\right) \mu_{2}^{4} /\left(4 \pi^{2}\right)=B$, the total energy density $\epsilon_{2}=3 P_{2}+B=4 B$, and the baryon number density $n_{B 2}=\left(n_{u}+n_{d}\right) / 3=\mu_{2}^{3} / \pi^{2}$, giving an energy per baryon of

$$
\epsilon_{2} / n_{B 2}=\left(1+2^{4 / 3}\right)^{3 / 4}\left(4 \pi^{2}\right)^{1 / 4} B^{1 / 4}=6.441 B^{1 / 4} \approx 934 \mathrm{MeV} B_{145}^{1 / 4},
$$

where $B_{145}^{1 / 4} \equiv B^{1 / 4} / 145 \mathrm{MeV} ; 145 \mathrm{MeV}$ being the lowest possible choice for reasons discussed below.

A three-flavor quark gas is electrically neutral for $n_{u}=n_{d}=n_{s}$, i. e. $\mu_{3} \equiv \mu_{u}=$ $\mu_{d}=\mu_{s}$. For fixed bag constant the three-quark gas should exert the same pressure as the two-quark gas (leaving also the energy density, $\epsilon_{3}=3 P_{3}+B=4 B$, unchanged). That happens when $\mu_{3}=\left[\left(1+2^{4 / 3}\right) / 3\right]^{1 / 4} \mu_{2}$, giving a baryon number density of $n_{B 3}=\mu_{3}^{3} / \pi^{2}=\left[\left(1+2^{4 / 3}\right) / 3\right]^{3 / 4} n_{B 2}$. The energy per baryon is then

$$
\epsilon_{3} / n_{B 3}=3 \mu_{3}=3^{3 / 4}\left(4 \pi^{2}\right)^{1 / 4} B^{1 / 4}=5.714 B^{1 / 4} \approx 829 \mathrm{MeV} B_{145}^{1 / 4} ;
$$

lower than in the two-quark case by a factor $n_{B 2} / n_{B 3}=\left(3 /\left(1+2^{4 / 3}\right)\right)^{3 / 4} \approx 0.89$.

The possible presence of electrons was neglected in the calculations above. For two-flavor quark matter, including electrons in chemical equilibrium via $u+e^{-} \leftrightarrow$ $d+\nu_{e}$, so that $\mu_{u}+\mu_{e}=\mu_{d}$, gives more cumbersome equations, but only changes $\epsilon_{2} / n_{B 2}$ to $6.445 B^{1 / 4}$, since $\mu_{e}$ turns out to be rather small. Three-flavor quark matter does not contain electrons for non-interacting, massless quarks.

One may therefore gain of order $100 \mathrm{MeV}$ per baryon by introducing an extra flavor. At fixed confining bag pressure the extra Fermi-well allows one to pack the baryon number denser into the system, thereby gaining in binding energy.

The energy per baryon in a free gas of neutrons is the neutron mass, $m_{n}=$ $939.6 \mathrm{MeV}$; in a gas of ${ }^{56} \mathrm{Fe}$ it is $930 \mathrm{MeV}$. Naively, stability of $u d$-quark matter relative to neutrons thus corresponds to $\epsilon_{2} / n_{B 2}<m_{n}$, or $B^{1 / 4}<145.9 \mathrm{MeV}\left(B^{1 / 4}<\right.$

\footnotetext{
${ }^{1}$ Since current quark masses rather than constituent quark masses enter in the MIT bag model used to describe SQM, this is a very good approximation for $u$ and $d$-quarks with $5 \mathrm{MeV} \approx m_{u}<$ $m_{d} \approx 10 \mathrm{MeV} \ll 300 \mathrm{MeV} \approx \mu_{u}, \mu_{d}$.
} 
144.4MeV for stability relative to iron). The argument can be turned around: Since one observes neutrons and ${ }^{56} \mathrm{Fe}$ in Nature, rather than $u d$-quark matter, it is concluded that $B^{1 / 4}$ must be larger than the numbers just quoted. More detailed calculations including finite-size effects and Coulomb-forces do not change these numbers much, so we shall assume for the present purpose that $B^{1 / 4}=145 \mathrm{MeV}$ is an experimental lower limit for $\alpha_{s}=0$. (Here $\alpha_{s}$ denotes the strong "fine-structure" constant; $\alpha_{s}=0$ corresponding to non-interacting quarks except for the confinement given by $B)$.

Bulk strange quark matter is absolutely stable relative to a gas of iron for $B^{1 / 4}<$ $162.8 \mathrm{MeV}$, metastable relative to a neutron gas for $B^{1 / 4}<164.4 \mathrm{MeV}$, and relative to a gas of $\Lambda$-particles (the ultimate production limit in heavy-ion collisions) for $B^{1 / 4}<195.2 \mathrm{MeV}$. These numbers are upper limits. As demonstrated below, a finite $s$-quark mass as well as a non-zero strong coupling constant decreases the limit on $B^{1 / 4}$.

The presence of ordinary nuclei in Nature cannot be used to turn the values of $B^{1 / 4}$ just quoted for SQM into lower limits. Conversion of a nucleus into a lump of SQM requires simultaneous transformation of roughly $A u$ - and $d$-quarks into $s$ quarks. The probability for this to happen involves a weak interaction coupling to the power $A$, i. e. it does not happen. This leads to the conclusion, that even if SQM is the lowest energy state for hadronic matter in bulk, its formation requires a strangeness-rich environment or formation via a "normal" quark-gluon plasma in relativistic heavy-ion collisions, the early Universe, or a neutron star interior. All of these possibilities will be explored in the following.

\subsection{SQM in bulk at $T=0$}

The estimates above assumed $m_{s}=\alpha_{s}=0$. Non-zero $\alpha_{s}$ was found by Farhi and Jaffe [16] to correspond effectively to a reduction in $B$. In the interest of simplicity I will therefore set $\alpha_{s}=0$ in most of the following. The energy "penalty" paid by having to form $s$-quarks at a finite mass of $50-300 \mathrm{MeV}$ calls for more detailed calculations, however. Such calculations are usually performed within the MIT bag model [17, 18].

Strange quark matter contains degenerate Fermi gases of $u, d$, and $s$ quarks, and $e^{-}$or $e^{+}$. Chemical equilibrium is maintained by weak interactions,

$$
\begin{aligned}
d & \leftrightarrow u+e^{-}+\bar{\nu}_{e} \\
s & \leftrightarrow u+e^{-}+\bar{\nu}_{e} \\
u+s & \leftrightarrow d+u,
\end{aligned}
$$

where the first two reactions should be understood to include also the various permutations of the involved particles.

Neutrinos generally escape the system, so we shall ascribe to them no chemical 
potential. Thus the chemical potentials in equilibrium are given by

$$
\mu_{d}=\mu_{s}=\mu_{u}+\mu_{e} .
$$

Knowing the chemical potentials one can calculate the thermodynamic potentials.

$$
\begin{aligned}
& \Omega_{e, V}=-\frac{\mu_{e}^{4}}{12 \pi^{2}} \\
& \Omega_{u, V}=-\frac{\mu_{u}^{4}}{4 \pi^{2}} \\
& \Omega_{d, V}=-\frac{\mu_{d}^{4}}{4 \pi^{2}} \\
& \Omega_{s, V}=-\frac{\mu_{s}^{4}}{4 \pi^{2}}\left(\left(1-\lambda^{2}\right)^{1 / 2}\left(1-\frac{5}{2} \lambda^{2}\right)+\frac{3}{2} \lambda^{4} \ln \frac{1+\left(1-\lambda^{2}\right)^{1 / 2}}{\lambda}\right),
\end{aligned}
$$

defining $\lambda \equiv m_{s} / \mu_{s}$.

Number densities are given by

$$
n_{i, V}=-\partial \Omega_{i, V} / \partial \mu_{i} ;
$$

i. e. $n_{e, V}=\mu_{e}^{3} / 3 \pi^{2}, n_{u, V}=\mu_{u}^{3} / \pi^{2}, n_{d, V}=\mu_{d}^{3} / \pi^{2}, n_{s, V}=\mu_{s}^{3}\left(1-\lambda^{2}\right)^{3 / 2} / \pi^{2}$. The total pressure is

$$
P=\sum_{i} P_{i}-B=-\sum_{i} \Omega_{i, V}-B=0,
$$

and charge neutrality requires

$$
\frac{2}{3} n_{u, V}-\frac{1}{3} n_{d, V}-\frac{1}{3} n_{s, V}-n_{e, V}=0 .
$$

The total energy density is

$$
\epsilon=\sum_{i}\left(\Omega_{i, V}+n_{i, V} \mu_{i}\right)+B,
$$

and the density of baryon number

$$
n_{B}=\frac{1}{3}\left(n_{u, V}+n_{d, V}+n_{s, V}\right) .
$$

Combining Eqs. (6) and (13) leaves only one independent chemical potential, which can be determined from the pressure balance, Eq. (12). Thus all parameters can be calculated for a given choice of $m_{s}$ and $B$. Results of such calculations are shown in Figure [1. Similar calculations were originally done by Farhi and Jaffe [16].

The calculations above assumed zero temperature and external pressure. Finite temperature and external pressure can be relevant in connection with cosmology (Section 4) and strange stars (Section 5) respectively, and also for strangelet creation in collision experiments. The relevant extensions of the formulae above will be given in Section 3.4. 


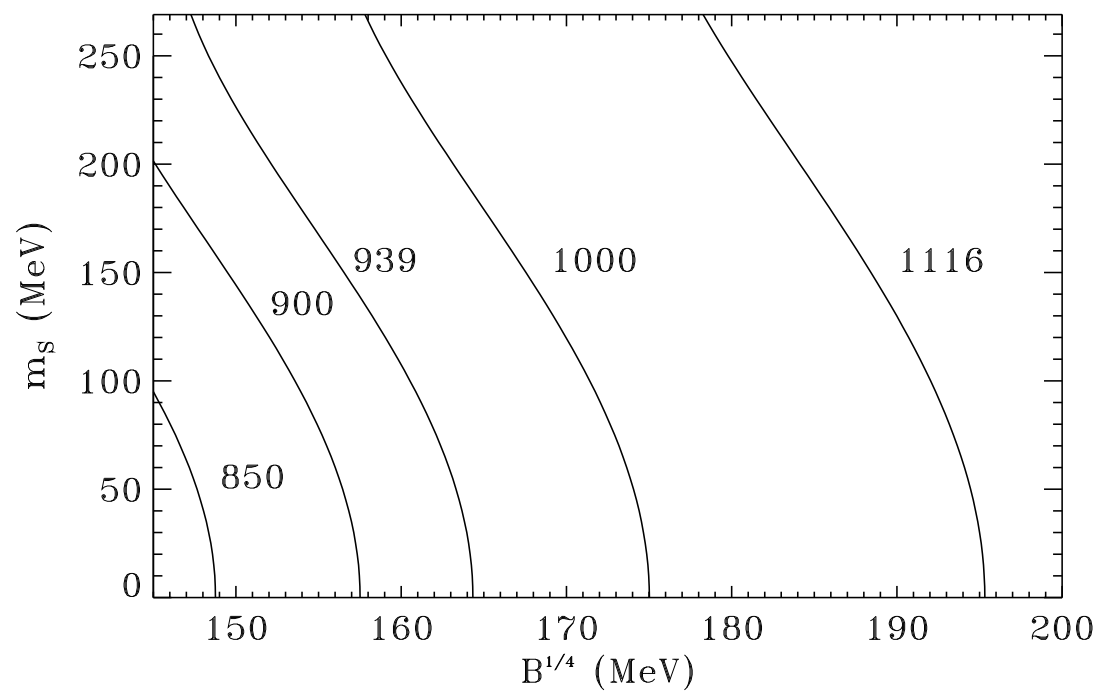

Figure 1: Energy per baryon for bulk strange quark matter as a function of bag constant and strange quark mass.

\section{Strangelets}

So far the treatment of SQM has focused on the bulk properties. This approximation is generally valid for large baryon numbers. For $A \ll 10^{7}$ the quark part of SQM is smaller than the Compton wavelength of electrons, so electrons no longer ensure local charge neutrality. Therefore Coulomb energy has to be taken into account, though the fortuitous cancellation of $q_{u}+q_{d}+q_{s}=\frac{2}{3}-\frac{1}{3}-\frac{1}{3}=0$ means that Coulomb energy is much less important for strangelets than for nuclei. For even smaller baryon numbers (in practice $A<10^{3}$ ) other finite size effects such as surface tension and curvature have to be taken into account.

Several strangelet searches with relativistic heavy-ion collisions as well as cosmic ray searches have been carried out, and others are planned for the future. Most of these searches are sensitive only to low $A$-values, so it is important to know the properties of small lumps of strange quark matter (strangelets).

In the following I will describe the physical properties of strangelets in the language of the MIT-bag model (only limited work has been performed using other models - qualitatively confirming the MIT-bag results, though quantitative details can differ). First, I will discuss results obtained from direct solution of the Dirac equation with MIT-bag boundary conditions; such mode filling calculations correspond to a nuclear shell model. Then I will show how the mean behavior of the shell model results can be understood physically in terms of a liquid drop model calculation based on a smoothed density of states, and how approximations to the liquid drop 
results give simple formulae for strangelet masses etc. Finally I discuss the changes introduced if strangelets are at finite rather than zero temperature.

\subsection{Shell model}

Mode-filling for large numbers of quarks in a spherical MIT-bag was performed for $u d$-systems by Vasak, Greiner and Neise [19], and for 2- and 3-flavor systems by Farhi and Jaffe [16], and Greiner et al. [20] (see also [21]). Gilson and Jaffe [22] published an investigation of low-mass strangelets for 4 different combinations of $s$ quark mass and bag constant with particular emphasis on metastability against strong decays. Further parameter ranges were studied and compared to liquid drop model calculations by Madsen [23], and recently new shell-model studies were published by Schaffner-Bielich et al. [24]. All of these calculations were performed for $\alpha_{s}=0$, which will also be assumed in the following.

In the MIT bag model noninteracting quarks are confined to a spherical cavity of radius $R$. They satisfy the free Dirac equation inside the cavity and obey a boundary condition at the surface, which corresponds to no current flow across the surface. The bag itself has an energy of $B V$. In the simplest version the energy (mass) of the system is given by the sum of the bag energy and the energies of individual quarks,

$$
E=\sum_{i=u, d, s} \sum_{\kappa} N_{\kappa, i}\left(m_{i}^{2}+k_{\kappa, i}^{2}\right)^{1 / 2}+B 4 \pi R^{3} / 3 .
$$

Here $k_{\kappa, i} \equiv x_{\kappa, i} / R$, where $x_{\kappa, i}$ are eigenvalues of the equation

$$
f_{\kappa}\left(x_{\kappa, i}\right)=\frac{-x_{\kappa, i}}{\left(x_{\kappa, i}^{2}+m_{i}^{2} R^{2}\right)^{1 / 2}+m_{i} R} f_{\kappa-1}\left(x_{\kappa, i}\right) .
$$

$f_{\kappa}$ are regular Bessel functions of order $\kappa$,

$$
f_{\kappa}(x)= \begin{cases}j_{\kappa}(x) & \kappa \geq 0 \\ y_{\kappa}(x)=(-1)^{\kappa+1} j_{-\kappa-1}(x) & \kappa<0\end{cases}
$$

For states with quantum numbers $(j, l) \kappa$ takes the values $\kappa= \pm\left(j+\frac{1}{2}\right)$ for $l=j \pm \frac{1}{2}$. For a given quark flavor each level has a degeneracy of $N_{\kappa, i}=3(2 j+1)$ (the factor 3 from color degrees of freedom). For example, the $1 S_{1 / 2}$ ground-state $(j=1 / 2, l=0$, $\kappa=-1$ ) for a massless quark corresponds to solving the equation $\tan x=x /(1-x)$, giving $x \simeq 2.0428$. The ground state has a degeneracy of 6 per flavor.

For massless quarks (finding the equilibrium radius from $\partial E / \partial R=0$ ) one gets

$$
E=364.00 \mathrm{MeV} B_{145}^{1 / 4}\left(\sum x_{\kappa, i}\right)^{3 / 4}
$$

where the sum is to be taken over all $3 A$ quark-levels, and the numbers $x_{\kappa, i}$ for massless quarks are tabulated in [19]. 
For massive quarks the level filling scheme is more cumbersome (see e.g. Refs. [22, 23]). Fixing bag constant and quark-masses, for each baryon number one must fill up the lowest energy levels for a choice of radius; then vary the radius until a minimum energy is found $(\partial E / \partial R=0)$. Since levels cross, the order of levels is changing as a function of $R$. This is easily seen in the Figures, where one notices discontinuous changes in the position of shells.
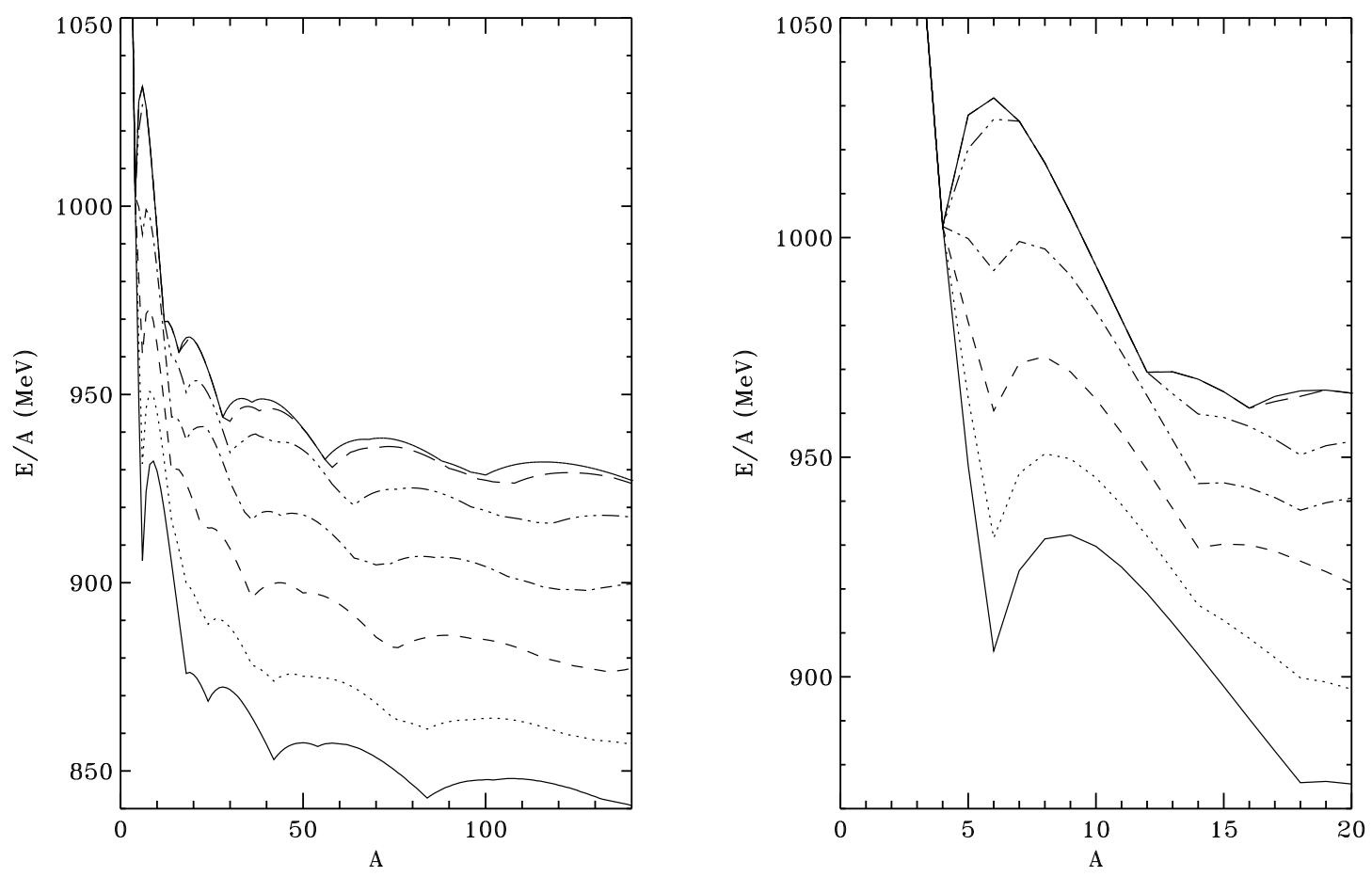

Figure 2: Energy per baryon (in $\mathrm{MeV}$ ) for strangelets with $B^{1 / 4}=145 \mathrm{MeV}$ and $m_{s}$ from $0-300 \mathrm{MeV}$ in steps of $50 \mathrm{MeV}$ ( $m_{s}$ increases upward). The figure on the right shows an expanded view of the low-mass region to highlight the change of "magic numbers" with changing $m_{s}$.

One notices that the energy per baryon smoothly approaches the bulk limit for $A \rightarrow \infty$, whereas the energy grows significantly for low $A$. For low $s$-quark mass shells are recognized for $A=6$ ( 3 colors and 2 spin orientations per flavor), and less conspicuous ones for $A=18,24,42,54,60,84,102$ etc. As $m_{s}$ increases it becomes more and more favorable to use $u$ and $d$ rather than $s$-quarks, and the "magic numbers" change; for instance the first closed shell is seen for $A=4$ rather than 6 .

Equation (16) can be modified by inclusion of Coulomb energy and zero-point fluctuation energy. As already discussed the Coulomb energy is generally small. 
The zero-point energy is normally included as a phenomenological term of the form $-Z_{0} / R$, where fits to light hadron spectra indicate the choice $Z_{0}=1.84$. This was used, for instance, by Gilson and Jaffe [22]. Roughly half of this phenomenological term is due to center-of-mass motion, which can be included more explicitly by substituting $\left[\left(\sum x_{\kappa, i}\right)^{2}-\sum x_{\kappa, i}^{2}\right]^{3 / 8}$ instead of $\left(\sum x_{\kappa, i}\right)^{3 / 4}$ in Eq. (19). The proper choice of $\alpha_{s}$ and $Z_{0}$ is a tricky question. As discussed by Farhi and Jaffe [16] the values are intimately coupled to $B$ and $m_{s}$, and it is not obvious that values deduced from bag model fits to ordinary hadrons are to be preferred. This uncertainty may have an important effect for $A<5$-10, but the zero-point energy quickly becomes negligible for increasing $A$ for reasons explained in Section 3.2.2. It means, however, that it is difficult to match strangelet calculations to experimental data concerning ordinary hadrons or limits on the putative $A=2 H$-dibaryon.

\subsection{Liquid drop model}

Mode-filling calculations are rather tedious but do of course give the "correct" results as far as the model can be trusted. But for many applications a global mass-formula analogous to the liquid drop model for nuclei is of great use and also gives further physical insight.

A phenomenological approach to a strangelet mass-formula was undertaken by Crawford et al. [25, 26] whereas Berger and Jaffe [27] made a detailed analysis within the MIT bag model. They included Coulomb corrections and surface tension effects stemming from the depletion in the surface density of states due to the mass of the strange quark. Both effects were treated as perturbations added to a bulk solution with the surface contribution derived from a multiple reflection expansion. Madsen [23, 28, 29] gave a self-consistent treatment including also the very important curvature energy.

The following discussion closely follows [23]. All calculations are done for zero temperature and strong coupling constant, $\alpha_{s}$. As argued by Farhi and Jaffe [16] the latter assumption can be relaxed by a re-scaling of the bag constant. Also, I shall concentrate on systems small enough $\left(A<10^{7}\right)$ to justify neglect of electrons. Strangelets with $A \ll 10^{7}$ are smaller than the electron Compton wavelength, and electrons are therefore mainly localized outside the quark phase. Thus strangelets do not obey a requirement of local charge neutrality, as was the case for SQM in bulk. This leads to a small Coulomb energy, which is rather negligible for the mass-formula (less than a few $\mathrm{MeV}$ per baryon), but which is decisive for the charge-to-mass ratio of the strangelet. A characteristic of strangelets, which is perhaps the best experimental signature, is that this ratio is very small compared to ordinary nuclei. Finally, I neglect charge screening, an issue of negligible importance for the mass formula, but

of some importance for the charge-to-mass ratio for systems of radii above 5-10 fm $\left(A>10^{2}-10^{3}\right)$ [30]. 
In the ideal Fermi-gas approximation the energy of a system composed of quark flavors $i$ is given by

$$
E=\sum_{i}\left(\Omega_{i}+N_{i} \mu_{i}\right)+B V+E_{\text {Coul }}
$$

Here $\Omega_{i}, N_{i}$ and $\mu_{i}$ denote thermodynamic potentials, total number of quarks, and chemical potentials, respectively. $B$ is the bag constant, $V$ is the bag volume, and $E_{\text {Coul }}$ is the Coulomb energy.

In the multiple reflection expansion framework of Balian and Bloch [31], the thermodynamical quantities can be derived from a density of states of the form

$$
\frac{d N_{i}}{d k}=6\left\{\frac{k^{2} V}{2 \pi^{2}}+f_{S}\left(\frac{m_{i}}{k}\right) k S+f_{C}\left(\frac{m_{i}}{k}\right) C+\ldots\right\}
$$

where area $S=\oint d S\left(=4 \pi R^{2}\right.$ for a sphere) and extrinsic curvature $C=\oint\left(\frac{1}{R_{1}}+\frac{1}{R_{2}}\right) d S$ (=8 $=8$ for a sphere). Curvature radii are denoted $R_{1}$ and $R_{2}$. For a spherical system $R_{1}=R_{2}=R$. The functions $f_{S}$ and $f_{C}$ will be discussed below.

In terms of volume-, surface-, and curvature-densities, $n_{i, V}, n_{i, S}$, and $n_{i, C}$, the number of quarks of flavor $i$ is

$$
N_{i}=\int_{0}^{k_{F i}} \frac{d N_{i}}{d k} d k=n_{i, V} V+n_{i, S} S+n_{i, C} C,
$$

with Fermi momentum $k_{F i}=\left(\mu_{i}^{2}-m_{i}^{2}\right)^{1 / 2}=\mu_{i}\left(1-\lambda_{i}^{2}\right)^{1 / 2} ; \lambda_{i} \equiv m_{i} / \mu_{i}$.

The corresponding thermodynamic potentials are related by

$$
\Omega_{i}=\Omega_{i, V} V+\Omega_{i, S} S+\Omega_{i, C} C,
$$

where $\partial \Omega_{i} / \partial \mu_{i}=-N_{i}$, and $\partial \Omega_{i, j} / \partial \mu_{i}=-n_{i, j}$. The volume terms are given by

$$
\begin{gathered}
\Omega_{i, V}=-\frac{\mu_{i}^{4}}{4 \pi^{2}}\left[\left(1-\lambda_{i}^{2}\right)^{1 / 2}\left(1-\frac{5}{2} \lambda_{i}^{2}\right)+\frac{3}{2} \lambda_{i}^{4} \ln \frac{1+\left(1-\lambda_{i}^{2}\right)^{1 / 2}}{\lambda_{i}}\right], \\
n_{i, V}=\frac{\mu_{i}^{3}}{\pi^{2}}\left(1-\lambda_{i}^{2}\right)^{3 / 2} .
\end{gathered}
$$

The surface contribution from massive quarks is derived from

$$
f_{S}\left(\frac{m}{k}\right)=-\frac{1}{8 \pi}\left\{1-\left(\frac{2}{\pi}\right) \tan ^{-1} \frac{k}{m}\right\}
$$

as 27

$$
\begin{aligned}
\Omega_{i, S}= & \frac{3}{4 \pi} \mu_{i}^{3}\left[\frac{\left(1-\lambda_{i}^{2}\right)}{6}-\frac{\lambda_{i}^{2}\left(1-\lambda_{i}\right)}{3}\right. \\
& \left.-\frac{1}{3 \pi}\left(\tan ^{-1}\left[\frac{\left(1-\lambda_{i}^{2}\right)^{1 / 2}}{\lambda_{i}}\right]-2 \lambda_{i}\left(1-\lambda_{i}^{2}\right)^{1 / 2}+\lambda_{i}^{3} \ln \left[\frac{1+\left(1-\lambda_{i}^{2}\right)^{1 / 2}}{\lambda_{i}}\right]\right)\right]
\end{aligned}
$$




$$
n_{i, S}=-\frac{3}{4 \pi} \mu_{i}^{2}\left[\frac{\left(1-\lambda_{i}^{2}\right)}{2}-\frac{1}{\pi}\left(\tan ^{-1}\left[\frac{\left(1-\lambda_{i}^{2}\right)^{1 / 2}}{\lambda_{i}}\right]-\lambda_{i}\left(1-\lambda_{i}^{2}\right)^{1 / 2}\right)\right] .
$$

For massless quarks $\Omega_{i, S}=n_{i, S}=0$, whereas $f_{C}(0)=-1 / 24 \pi^{2}$ gives [16, 28, 29] $\Omega_{i, C}=\mu_{i}^{2} / 8 \pi^{2} ; n_{i, C}=-\mu_{i} / 4 \pi^{2}$.

The curvature terms have never been derived for massive quarks, but as shown by Madsen [23], the following Ansatz (found from analogies with the surface term and other known cases) works:

$$
f_{C}\left(\frac{m}{k}\right)=\frac{1}{12 \pi^{2}}\left\{1-\frac{3}{2} \frac{k}{m}\left(\frac{\pi}{2}-\tan ^{-1} \frac{k}{m}\right)\right\} .
$$

This expression has the right limit for massless quarks $\left(f_{C}=-1 / 24 \pi^{2}\right)$ and for infinite mass, which corresponds to the Dirichlet boundary conditions studied by Balian and Bloch [31] $\left(f_{C}=1 / 12 \pi^{2}\right)$. Furthermore, the expression gives perfect fits to mode-filling calculations (see the Figures and discussion below). From this Ansatz one derives the following thermodynamical potential and density:

$$
\begin{gathered}
\Omega_{i, C}=\frac{\mu_{i}^{2}}{8 \pi^{2}}\left[\lambda_{i}^{2} \log \frac{1+\left(1-\lambda_{i}^{2}\right)^{1 / 2}}{\lambda_{i}}+\frac{\pi}{2 \lambda_{i}}-\frac{3 \pi \lambda_{i}}{2}+\pi \lambda_{i}^{2}-\frac{1}{\lambda_{i}} \tan ^{-1} \frac{\left(1-\lambda_{i}^{2}\right)^{1 / 2}}{\lambda_{i}}\right] \\
n_{i, C}=\frac{\mu_{i}}{8 \pi^{2}}\left[\left(1-\lambda_{i}^{2}\right)^{1 / 2}-\frac{3 \pi}{2} \frac{\left(1-\lambda_{i}^{2}\right)}{\lambda_{i}}+\frac{3}{\lambda_{i}} \tan ^{-1} \frac{\left(1-\lambda_{i}^{2}\right)^{1 / 2}}{\lambda_{i}}\right] .
\end{gathered}
$$

With these prescriptions the differential of $E\left(V, S, C, N_{i}\right)$ is given by

$$
d E=\sum_{i}\left(\Omega_{i, V} d V+\Omega_{i, S} d S+\Omega_{i, C} d C+\mu_{i} d N_{i}\right)+B d V+d E_{\text {Coul }}
$$

Minimizing the total energy at fixed $N_{i}$ by taking $d E=0$ for a sphere gives the pressure equilibrium constraint

$$
B=-\sum_{i} \Omega_{i, V}-\frac{2}{R} \sum_{i} \Omega_{i, S}-\frac{2}{R^{2}} \sum_{i} \Omega_{i, C}-\frac{d E_{\mathrm{Coul}}}{d V}
$$

with

$$
\begin{gathered}
E_{\text {Coul }}=\frac{\alpha Z_{V}^{2}}{10 R}+\frac{\alpha Z^{2}}{2 R}, \\
\frac{d E_{\text {Coul }}}{d V}=-\frac{\alpha Z_{V}^{2}}{40 \pi R^{4}}-\frac{\alpha Z^{2}}{8 \pi R^{4}},
\end{gathered}
$$

where $Z_{V}=\sum_{i} q_{i} n_{i, V} V$ is the volume part of the total charge, $Z$, whereas charge $Z-Z_{V}=\sum_{i} q_{i}\left(n_{i, S} S+n_{i, C} C\right)$ is distributed on the surface. The quark charges are 
$q_{u}=2 / 3, q_{d}=q_{s}=-1 / 3$. Eliminating $B$ from Eq. (20) then gives the energy for a spherical quark lump as

$$
E=\sum_{i}\left(N_{i} \mu_{i}+\frac{1}{3} \Omega_{i, S} S+\frac{2}{3} \Omega_{i, C} C\right)+\frac{4}{3} E_{\text {Coul }} .
$$

The optimal composition for fixed baryon number, $A$, can be found by minimizing the energy with respect to $N_{i}$ at fixed $V, S$, and $C$ giving

$$
0=d E=\sum_{i}\left(\mu_{i}+\frac{\partial E_{\text {Coul }}}{\partial N_{i}}\right) d N_{i}
$$

\subsubsection{Massless quarks-Bulk limit}

For uncharged bulk quark matter Eq. (36) reduces to the usual result for the energy per baryon

$$
\epsilon^{0}=A^{-1} \sum_{i} N_{i}^{0} \mu_{i}^{0}
$$

where superscript 0 denotes bulk values. The energy minimization, Eq. (33), corresponds to

$$
B=-\sum_{i} \Omega_{i, V}^{0}=\sum_{i} \frac{\left(\mu_{i}^{0}\right)^{4}}{4 \pi^{2}} .
$$

The last equality assumes massless quarks. In the bulk limit the baryon number density is given by

$$
n_{A}^{0}=\frac{1}{3} \sum_{i} \frac{\left(\mu_{i}^{0}\right)^{3}}{\pi^{2}}
$$

and one may define a bulk radius per baryon as

$$
R^{0}=\left(3 / 4 \pi n_{A}^{0}\right)^{1 / 3}
$$

For quark matter composed of massless $u, d$, and $s$-quarks, the Coulomb energy vanishes at equal number densities due to the fact that the sum of the quark charges is zero. Thus it is energetically most favorable to have equal chemical potentials for the three flavors. From the equations above one may derive the following bulk expressions for 3-flavor quark matter:

$$
\begin{gathered}
\mu_{i}^{0}=\left(\frac{4 \pi^{2} B}{3}\right)^{1 / 4}=1.905 B^{1 / 4}=276.2 \mathrm{MeV}_{145}^{1 / 4} \\
n_{A}^{0}=\left(\mu_{i}^{0}\right)^{3} / \pi^{2}=0.700 B^{3 / 4} \\
R^{0}=\left(3 / 4 \pi n_{A}^{0}\right)^{1 / 3}=0.699 B^{-1 / 4}
\end{gathered}
$$


And the energy per baryon is

$$
\epsilon^{0}=3 \mu_{i}^{0}=5.714 B^{1 / 4}
$$

in agreement with Eq. (2)).

Following Berger and Jaffe [27] one may to first order regard Coulomb, surface (and here correspondingly curvature) energies as perturbations on top of the bulk solution. In this approach one gets

$$
\begin{aligned}
\frac{E}{A} & =\epsilon^{0}+A^{-1} \sum_{i} \Omega_{i, C}^{0} C^{0}=\epsilon^{0}+\frac{3^{13 / 12} B^{1 / 4}}{\pi^{1 / 6} 2^{1 / 6} A^{2 / 3}} \\
& \approx\left[829 \mathrm{MeV}+351 \mathrm{MeV} A^{-2 / 3}\right] B_{145}^{1 / 4} .
\end{aligned}
$$

The corresponding result for 2-flavor quark matter (c.f. [29]) is

$$
\frac{E}{A}=\epsilon^{0}+A^{-1} \sum_{i} \Omega_{i, C}^{0} C^{0} \approx\left[934 \mathrm{MeV}+291 \mathrm{MeV} A^{-2 / 3}\right] B_{145}^{1 / 4} .
$$

\subsubsection{Massive s-quarks-Bulk limit}

For $m_{s}>0$ the energy minimization, Eq. (39), changes to

$$
\begin{aligned}
B & =-\sum_{i} \Omega_{i, V}^{0} \\
& =\sum_{i=u, d} \frac{\left(\mu_{i}^{0}\right)^{4}}{4 \pi^{2}}+\frac{\left(\mu_{s}^{0}\right)^{4}}{4 \pi^{2}}\left[\left(1-\lambda^{2}\right)^{1 / 2}\left(1-\frac{5}{2} \lambda^{2}\right)+\frac{3}{2} \lambda^{4} \ln \frac{1+\left(1-\lambda^{2}\right)^{1 / 2}}{\lambda}\right],
\end{aligned}
$$

and the baryon number density is now given by

$$
n_{A}^{0}=\frac{1}{3}\left[\sum_{i=u, d} \frac{\left(\mu_{i}^{0}\right)^{3}}{\pi^{2}}+\frac{\left(\mu_{s}^{0}\right)^{3}}{\pi^{2}}\left(1-\lambda^{2}\right)^{3 / 2}\right] .
$$

A bulk radius per baryon is still defined by Eq. (41).

In bulk equilibrium the chemical potentials of the three quark flavors are equal, $\mu_{u}^{0}=\mu_{d}^{0}=\mu_{s}^{0} \equiv \mu^{0}=\epsilon^{0} / 3$. Neglecting Coulomb energy one may approximate the energy per baryon of small strangelets as a sum of bulk, surface and curvature terms, using the chemical potential calculated in bulk:

$$
\frac{E}{A}=\epsilon^{0}+A^{-1} \sum_{i} \Omega_{i, S}^{0} S^{0}+A^{-1} \sum_{i} \Omega_{i, C}^{0} C^{0},
$$

where $S^{0}=4 \pi\left(R^{0}\right)^{2} A^{2 / 3}$ and $C^{0}=8 \pi\left(R^{0}\right) A^{1 / 3}$. Examples for $B^{1 / 4}=145 \mathrm{MeV}$ are (with $s$-quark mass in $\mathrm{MeV}$ given in parenthesis) 


$$
\begin{aligned}
\epsilon(0) & =829 \mathrm{MeV}+0 \mathrm{MeV} A^{-1 / 3}+351 \mathrm{MeV} A^{-2 / 3} \\
\epsilon(50) & =835 \mathrm{MeV}+61 \mathrm{MeV} A^{-1 / 3}+277 \mathrm{MeV} A^{-2 / 3} \\
\epsilon(150) & =874 \mathrm{MeV}+77 \mathrm{MeV} A^{-1 / 3}+232 \mathrm{MeV} A^{-2 / 3} \\
\epsilon(200) & =896 \mathrm{MeV}+53 \mathrm{MeV} A^{-1 / 3}+242 \mathrm{MeV} A^{-2 / 3} \\
\epsilon(250) & =911 \mathrm{MeV}+22 \mathrm{MeV} A^{-1 / 3}+266 \mathrm{MeV} A^{-2 / 3} \\
\epsilon(300) & =917 \mathrm{MeV}+0.3 \mathrm{MeV} A^{-1 / 3}+295 \mathrm{MeV} A^{-2 / 3} \\
\epsilon(350) & =917 \mathrm{MeV}+0 \mathrm{MeV} A^{-1 / 3}+296 \mathrm{MeV} A^{-2 / 3}
\end{aligned}
$$

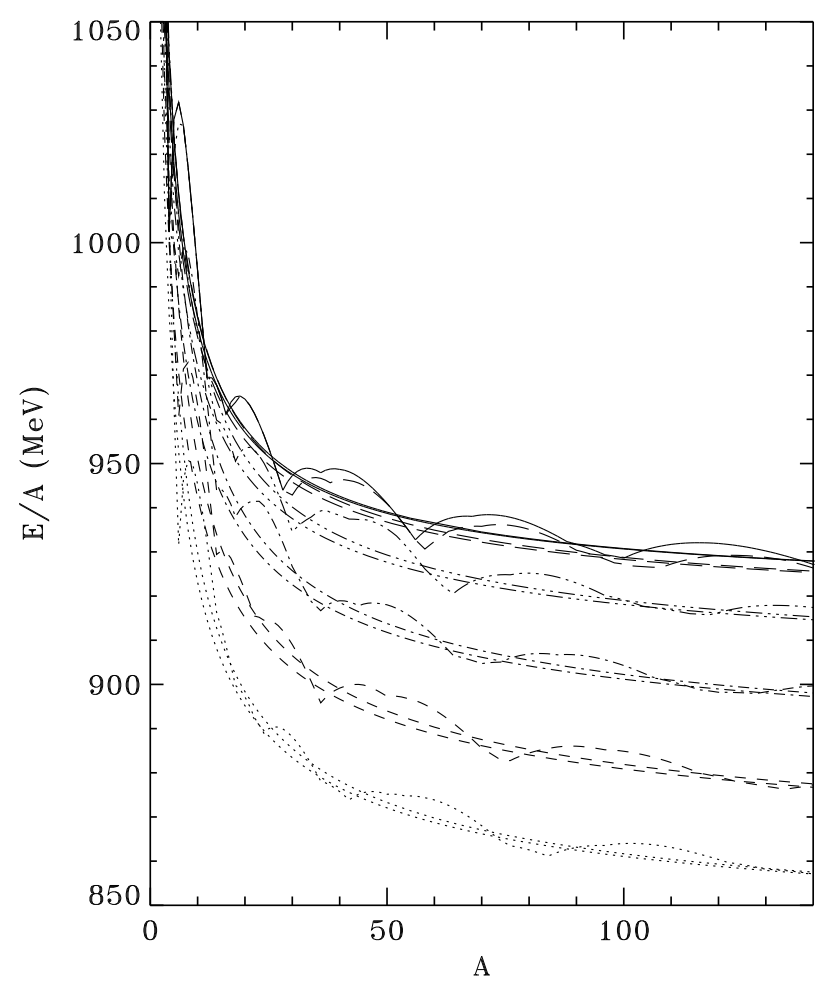

Figure 3: Shell-model and liquid drop model results compared for $B^{1 / 4}=145 \mathrm{MeV}$ with massless $u$ and $d$ quarks, and with $m_{s}$ in the range $50-300 \mathrm{MeV}$ in steps of $50 \mathrm{MeV}$. For each value of $m_{s}$ the upper smooth curve is the full liquid drop model result, whereas the lower smooth curve is the bulk approximation.

The bulk approximations above generally undershoot the correct solution with properly smoothed density of states by $2 \mathrm{MeV}$ for $A>100,5 \mathrm{MeV}$ for $A \approx 50,10 \mathrm{MeV}$ for $A \approx 10$ and $20 \mathrm{MeV}$ for $A \approx 5$ (Figure 3). This is because the actual chemical 
potentials of the quarks increase when $A$ decreases, whereas the bulk approximations use constant $\mu$. For massless $s$-quarks the expression for $\epsilon(0)$ scales simply as $B^{1 / 4}$. The same scaling applies for $m_{s}>\epsilon^{0} / 3$, where no $s$-quarks are present; in the example above the scaling can be applied to $\epsilon(350)$. For intermediate $s$-quark masses both $\epsilon$ and $m_{s}$ should be multiplied by $B_{145}^{1 / 4}$ to scale the results. For instance, if $B^{1 / 4}=165 \mathrm{MeV}$ one finds $\epsilon(150)=985 \mathrm{MeV}+93 \mathrm{MeV} A^{-1 / 3}+265 \mathrm{MeV}^{-2 / 3}$; $\epsilon(250)=1027 \mathrm{MeV}+46 \mathrm{MeV} A^{-1 / 3}+284 \mathrm{MeV}^{-2 / 3}$. Coulomb effects were not included above. Their inclusion would have no influence for $m_{s} \rightarrow 0$, but would change the results by a few $\mathrm{MeV}$ for large $m_{s}$. In particular charge neutral $u d-$ quark matter has $\epsilon=\left[934 \mathrm{MeV}+291 \mathrm{MeV} A^{-2 / 3}\right] B_{145}^{1 / 4}$ (Eq. (47)) rather than the $\left[917 \mathrm{MeV}+296 \mathrm{MeV} A^{-2 / 3}\right] B_{145}^{1 / 4}$ found above (Eq. (57)).

In connection with the shell-model calculations I described the effects of a zeropoint energy of the form $-Z_{0} / R$, and claimed that it was important only for $A<10$. This can be understood in the bulk approximation of constant $\mu$, because the zeropoint term per baryon is proportional to $A^{-4 / 3}$ compared to $A^{-1 / 3}$ and $A^{-2 / 3}$ for surface and curvature energies. The full term to be added to the bulk approximation expressions for a given $\epsilon^{0}$ is:

$$
\epsilon_{\text {zero }}=-Z_{0}(4 / 243 \pi)^{1 / 3}\left[2+\left[1-\left(3 m_{s} / \epsilon^{0}\right)\right]^{3 / 2}\right]^{1 / 3} \epsilon^{0} A^{-4 / 3}
$$

typically of order $-200 Z_{0} \mathrm{MeV} A^{-4 / 3}$.

\subsection{Shell model versus liquid drop model}

Self-consistent solutions can be obtained from Eq. (36). These solutions are compared to the shell-model calculations and the bulk approximations in the Figures. The fits are very good, showing that inclusion of surface tension and curvature energy via the multiple reflection expansion explains the overall behavior of the results.

3-flavor quark matter is energetically favored in bulk, and could be absolutely stable relative to ${ }^{56} \mathrm{Fe}$ for $144 \mathrm{MeV}<B^{1 / 4}<163 \mathrm{MeV}$. The lower limit corresponds to experimentally excluded stability of $u d$ quark matter, whereas the upper limit corresponds to a bulk energy per baryon of $u d s$-matter of $930 \mathrm{MeV}$ for $m_{s}=0$.

Finite-size systems are strongly destabilized by the curvature energy, with a magnitude of about $300 \mathrm{MeV} A^{-2 / 3} B_{145}^{1 / 4}$ for 3 quark flavors. This may pose problems for the experimental attempts of producing strange quark matter, since these experiments so far can only hope to create quark lumps with baryon number $A<20-30$, and observe lifetimes exceeding $10^{-8}$ seconds. Further destabilization occurs for finitemass $s$-quarks, where the surface tension (exactly zero for massless quarks) adds up to $90 \mathrm{MeV} A^{-1 / 3}$ to the energy.

Writing $E / A=\epsilon^{0}+c_{\mathrm{surf}} A^{-1 / 3}+c_{\mathrm{curv}} A^{-2 / 3}$, with $c_{\text {surf }} \approx 100 \mathrm{MeV}$ and $c_{\mathrm{curv}} \approx$ 

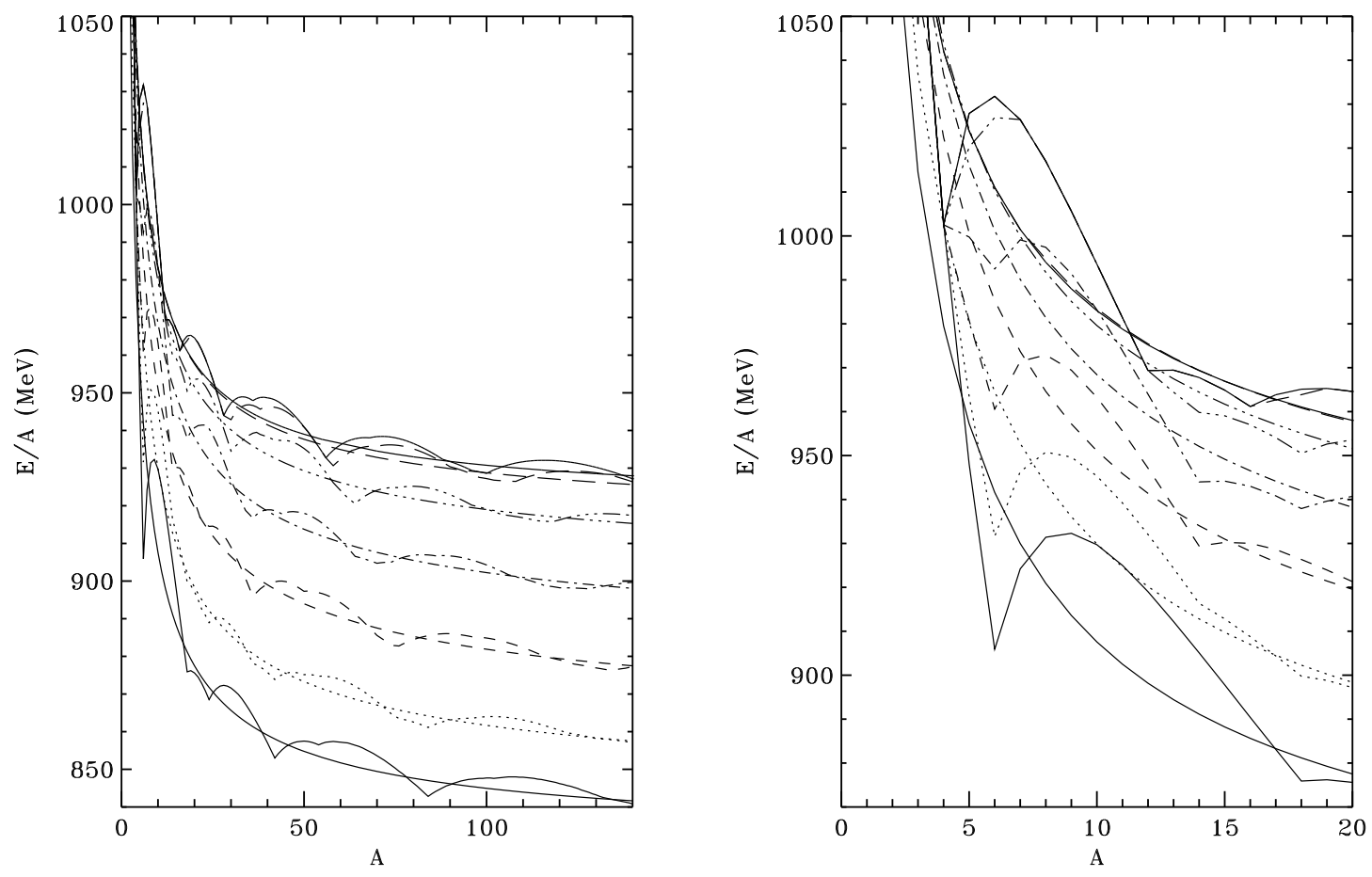

Figure 4: As Figure 2 but showing also the liquid drop results.

$300 \mathrm{MeV}$, the stability condition $E / A<m_{n}$ may be written as $A>A_{\text {min }}^{\text {abs }}$, where

$$
A_{\mathrm{min}}^{\mathrm{abs}}=\left(\frac{c_{\mathrm{surf}}+\left[c_{\mathrm{surf}}^{2}+4 c_{\mathrm{curv}}\left(m_{n}-\epsilon^{0}\right)\right]^{1 / 2}}{2\left(m_{n}-\epsilon^{0}\right)}\right)^{3} .
$$

Stability at baryon number 30 requires a bulk binding energy in excess of $65 \mathrm{MeV}$, which is barely within reach for $m_{s}>100 \mathrm{MeV}$ if, at the same time, ud-quark matter shall be unstable. The proposed cosmic ray strangelet-candidates with baryon number 370 [32] would for stability require a bulk binding energy per baryon exceeding $20 \mathrm{MeV}$ to overcome the combined curvature and surface energies. Absolute stability relative to a gas of ${ }^{56} \mathrm{Fe}$ corresponds to furthermore using $930 \mathrm{MeV}$ instead of $m_{n}$, whereas stability relative to a gas of $\Lambda$-particles (the ultimate limit for formation of short-lived strangelets) would correspond to substitution of $m_{\Lambda}=1116 \mathrm{MeV}$.

Another way of stating the results is to calculate the minimum baryon number for which long-lived metastability with respect to neutron emission is possible. This requires $d E_{\text {curv }} / d A+d E_{\text {surf }} / d A<m_{n}-\epsilon^{0}$, or

$$
A_{\mathrm{min}}^{\mathrm{meta}}=\left(\frac{c_{\mathrm{surf}}+\left[c_{\mathrm{surf}}^{2}+3 c_{\mathrm{curv}}\left(m_{n}-\epsilon^{0}\right)\right]^{1 / 2}}{3\left(m_{n}-\epsilon^{0}\right)}\right)^{3} .
$$


To have $A_{\text {min }}^{\text {meta }}<30$ requires $m_{n}-\epsilon^{0}>30 \mathrm{MeV}$, which is possible, but only for a narrow range of parameters.

This should not, however, defer experimentalists from pursuing the proposed searches. After all, the MIT bag model is only an approximation, and in particular shell effects can have a stabilizing effect. As stressed by Gilson and Jaffe [22] the fact that the slope of $E / A$ versus $A$ becomes very steep near magic numbers can lead to strangelets that are metastable (stable against single baryon emission) even for $\epsilon^{0}>930 \mathrm{MeV}$. Also, the time-scale for energetically allowed decays has not been calculated. Pauli-blocking is known to delay weak quark conversion in strangelets [33, 34, 35, 36], and this will probably have a significant influence on the lifetimes. The existence of small baryon number strangelets is ultimately an experimental issue.

\subsection{Strangelets at finite temperature}

Whereas the calculations above deal with strangelets at zero temperature, the environment in heavy ion collisions is expected to be hot. An advantage of the asymptotic mass formula compared to the shell-model calculations is, that it can fairly easily be generalized to non-zero temperature.

The general expression for the thermodynamic potential, $\Omega_{i}$, is

$$
\Omega_{i}=\mp g_{i} T \int_{0}^{\infty} d k \frac{d N_{i}}{d k} \ln [1 \pm \exp (-(\epsilon(k)-\mu) / T)]
$$

where the upper sign is for fermions, the lower for bosons, and the density of states,

$\frac{d N_{i}}{d k}$, is given by Eq. (21). For massless quarks (including antiquarks) an integration gives, per flavor,

$$
\Omega_{q}=-\left(\frac{7 \pi^{2}}{60} T^{4}+\frac{\mu^{2} T^{2}}{2}+\frac{\mu^{4}}{4 \pi^{2}}\right) V+\left(\frac{T^{2}}{24}+\frac{\mu^{2}}{8 \pi^{2}}\right) C,
$$

with a corresponding quark number

$$
N_{q}=-\frac{\partial \Omega_{q}}{\partial \mu}=\left(\mu T^{2}+\frac{\mu^{3}}{\pi^{2}}\right) V-\frac{\mu}{4 \pi^{2}} C .
$$

For gluons

$$
\Omega_{g}=-\frac{8 \pi^{2}}{45} T^{4} V+\frac{4}{9} T^{2} C
$$

The total $\Omega$ can be found from summing the terms above, and other thermodynamical quantities like the free energy and the internal energy can be derived. For 3 massless quark flavors of equal chemical potential one finds

$$
\Omega=\left(-\frac{19 \pi^{2}}{36} T^{4}-\frac{3}{2} \mu^{2} T^{2}-\frac{3}{4 \pi^{2}} \mu^{4}+B\right) V+\left(\frac{41}{72} T^{2}+\frac{3}{8 \pi^{2}} \mu^{2}\right) C
$$




$$
\begin{aligned}
& F=\left(-\frac{19 \pi^{2}}{36} T^{4}+\frac{3}{2} \mu^{2} T^{2}+\frac{9}{4 \pi^{2}} \mu^{4}+B\right) V+\left(\frac{41}{72} T^{2}-\frac{3}{8 \pi^{2}} \mu^{2}\right) C \\
& E=\left(\frac{19 \pi^{2}}{12} T^{4}+\frac{9}{2} \mu^{2} T^{2}+\frac{9}{4 \pi^{2}} \mu^{4}+B\right) V-\left(\frac{41}{72} T^{2}+\frac{3}{8 \pi^{2}} \mu^{2}\right) C
\end{aligned}
$$

Strangelets are in mechanical equilibrium at fixed temperature and baryon number when $d F=0$, corresponding to

$$
B V=\left(\frac{19 \pi^{2}}{36} T^{4}+\frac{3}{2} \mu^{2} T^{2}+\frac{3}{4 \pi^{2}} \mu^{4}\right) V-\left(\frac{41}{216} T^{2}+\frac{1}{8 \pi^{2}} \mu^{2}\right) C
$$

In this case one gets the following expressions for the thermodynamic potential, free energy, internal energy and baryon number:

$$
\begin{gathered}
\Omega=\left(\frac{41}{108} T^{2}+\frac{1}{4 \pi^{2}} \mu^{2}\right) C \\
F=\left(3 \mu^{2} T^{2}+\frac{3}{\pi^{2}} \mu^{4}\right) V+\left(\frac{41}{108} T^{2}-\frac{1}{2 \pi^{2}} \mu^{2}\right) C \\
E=4 B V \\
A=\left(\mu T^{2}+\frac{1}{\pi^{2}} \mu^{3}\right) V-\frac{\mu}{4 \pi^{2}} C .
\end{gathered}
$$

Notice that the equations above can also be used in connection with bulk SQM, for instance in an astrophysical context, by simply putting $C=0$. An external pressure can be accommodated by substituting $B+P_{\text {external }}$ in place of $B$.

Dotted curves in Figure 5 shows the energy per baryon for finite temperature strangelets according to the formulae above. Results are given for fixed entropy per baryon, where the entropy is calculated from $S \equiv-\partial \Omega /\left.\partial T\right|_{V, \mu}$. These results were first presented in [37]. A similar treatment, including finite $m_{s}$, was published in 38], whereas Ref. [39] shows results for a corresponding finite temperature shell model calculation, finding that shell structures are washed away at $T>10 \mathrm{MeV}$, which means that liquid drop model and shell model results become indistinguishable at high $T(S / A)$.

As discussed in more detail in [37] further complications arise from the fact, that strangelets must be color singlets. This has no influence on the ground state energy for $T=0$, but for $T>0$ quarks are statistically distributed over energy levels, and the color singlet constraint reduces the number of possible configurations, forcing the energy up for fixed entropy (see also [40]). The effect is important for $A<100$ as illustrated in Figure 5. Similar effects result from insisting that strangelets shall have a definite momentum. These destabilising effects can be important in connection with experiments, which inevitably create strangelets with rather high entropies. A tremendous job remains to be done in calculating the details of strangelet formation, evolution, and decay modes, including realistic non-equilibrium effects, etc.! 


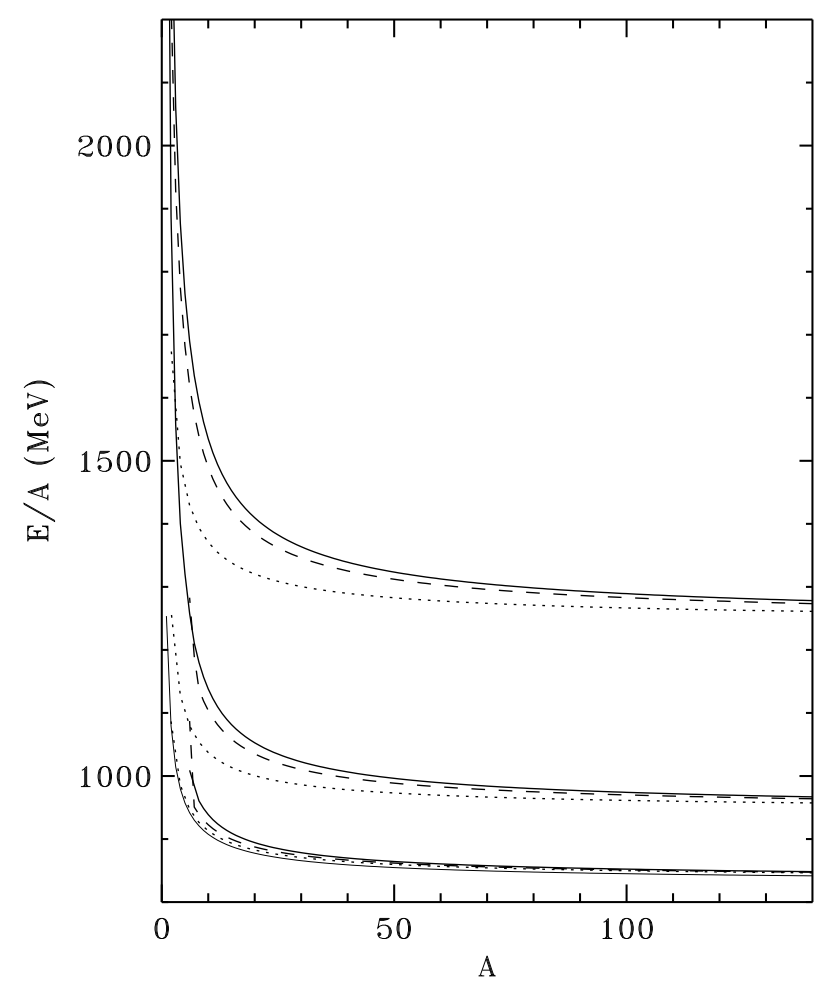

Figure 5: $E / A$ as a function of $A$ for strangelets with equal numbers of massless $u$, $d$, and $s$ quarks for entropy per baryon of $0,1,5$ and 10 , and $B^{1 / 4}=145 \mathrm{MeV}$. Solid curves include color singlet and zero momentum constraints, dashed curves only the color singlet constraint, and dotted curves are without constraints. Entropy increases upward. For $S=0(T=0)$ the three curves completely overlap (lowest solid curve).

\section{SQM in cosmology}

\subsection{Formation, evaporation and boiling of quark nuggets}

If the cosmological quark-hadron phase transition was first order, supercooling may result in concentration of baryon number inside shrinking bubbles of quark phase. The amount of baryon concentration depends on the permeability of the "membrane" separating the phases and on the turbulent removal of quarks from the phase boundary. If a quark bubble is able to get rid of entropy fast enough (primarily in the form of neutrinos and photons) relative to the rate of baryon number removal, there is a chance of reaching baryon number densities in the quark bubbles approaching nuclear matter density. In other words, a quark nugget may form. Whether or not this actually happens, or whether one is left with the less extreme, but also interesting 
scenario where all of the quarks end up in inhomogeneously distributed neutrons and protons, giving non-standard Big Bang nucleosynthesis, has been a topic of much debate [2, 41, 42, 43, 44], and the final word has probably not been said.

But even if cosmological quark nuggets do form, they find themselves in a very hostile environment with a temperature of order $100 \mathrm{MeV}$. Under such conditions the nuggets are unstable against surface evaporation 45, 46, 47, 48 and boiling [49, 50, 51]; but the crucial question from a cosmological point of view is whether some nuggets may survive due to the relatively short time-scale for cooling the Universe (the age of the Universe at temperature $T$ being roughly $t_{\mathrm{sec}}=T_{\mathrm{MeV}}^{-2}$ ).

Alcock and Farhi [45] showed that the timescale for complete evaporation of a quark nugget was smaller than the age of the Universe at temperature $T$ for baryon numbers below

$$
A_{\text {evap }} \approx 2 \times 10^{56} \exp \left(-3 I_{n} / T\right) f_{n}^{3}
$$

where $f_{n}$ (the phase boundary penetrability of neutrons) was assumed to be close to unity. For a homogeneous quark nugget the neutron binding energy $I_{n}=m_{n}-\mu_{u}-2 \mu_{d}$ was estimated to be of order $20 \mathrm{MeV}$. For such a binding, primordial nuggets with baryon number $A<10^{55}$ evaporate almost instantly when neutrino heating becomes possible at $T \approx 50 \mathrm{MeV} 45$.

However, the surface evaporation of neutrons and protons reduces $\mu_{u}$ and $\mu_{d}$, and leads to an increase in $\mu_{s}$. Weak decays, diffusion and convection work to counteract this, but the net result is an $s$-quark enriched layer near the surface. (Small nuggets are $s$-quark enriched throughout their interior). The most efficient way to remove the $s$-quarks is then to emit them in kaons $\left(\overline{K^{0}}, K^{-}\right)$along with thermal $\bar{u}$ and $\bar{d}$. A quasi-equilibrium situation arises with an effective $I_{n} \approx 350 \mathrm{MeV}$ 46. Thereby the baryon number of nuggets surviving evaporation is reduced to $10^{46}$, and a proper inclusion of reabsorption of emitted hadrons (a calculation that has so far not been done) may reduce the number somewhat.

Cosmological nugget evaporation (time-reversed) is closely related to the distillation mechanism proposed for strangelet production in relativistic heavy-ion collisions [52, 53, 20, 14, 54, 55, 56, 57]. There strangeness enhancement occurs due to emission of $K^{+}$and $K^{0}$.

The calculations described above assume that the penetrability of the phase boundary is near $100 \%$. It has been argued that the penetrability may be reduced by a few orders of magnitude in a chromoelectric flux tube model. This would decrease $A_{\text {evap }}$ by a factor $f_{n}^{3}$, permitting smaller nuggets (possibly down to $A=10^{39}$ ) to survive [47, 48]. Again, the limit on $A$ may be further reduced by reabsorption.

Primordial nuggets are superheated, and may therefore boil by forming bubbles of hadronic gas in their interiors 49 . However, even though boiling is thermodynamically allowed, it probably does not play an important role for primordial nuggets (or in heavy-ion collisions for that matter), since the time-scale is too short for bubblenucleation to take place [50, 51]. The surface evaporation described above is thus the 
decisive mechanism.

Some authors have argued [58, 59], that boiling will take place unless a large external pressure (e.g. due to a gravitationally bound shell of nucleons) is there to prevent it. Such gravitational stabilization only works for masses close to those of stars $\left(A \approx 10^{57}\right)$. However, the authors discuss only whether boiling is thermodynamically possible, but neglect that there is not enough time for the bubbles to nucleate.

Apart from trace abundances, one should not expect nuggets smaller than $10^{30}-$ $10^{40}$ to survive from the early Universe. This however brings one well within the causality limit set by the baryon number inside the horizon during the cosmic quarkhadron phase transition,

$$
A_{\mathrm{hor}} \approx 10^{49}\left(\frac{100 \mathrm{MeV}}{T}\right)^{2}
$$

and includes the "most probable" range of baryon numbers originally predicted by Witten [2]. It also leaves open the possibility that SQM may explain the dark matter problem, and if we understood the details of the quark-hadron phase transition, we could even calculate the relative abundances of dark and ordinary matter from first principles.

There is a possibility, that also small traces of primordial nuggets with low baryon numbers are left over from the early Universe. Even such traces may in fact be "observed" using the astrophysical detectors discussed in Section 6, or via Big Bang nucleosynthesis, as explained in Section 4.2.

\subsection{Quark nuggets and Big Bang nucleosynthesis}

A crucial property of quark nuggets is the positive electrostatic surface potential of the quark phase, which is due to the quarks being stronger bound than the electrons (electrostatic forces are weaker than strong forces). For typical nugget parameters the electrostatic potential can be several $\mathrm{MeV}$, so except at very high temperatures, protons and nuclei are repelled from nuggets, whereas neutrons are absorbed, adding one unit of baryon number.

This opens the intriguing possibility of using SQM as an energy source [14], at least in principle. It also makes it possible to use Big Bang nucleosynthesis as well as the properties of pulsars to place very stringent limits on the abundance of quark nuggets in the Universe.

During Big Bang nucleosynthesis $(T \leq 1 \mathrm{MeV})$, nuggets absorb neutrons but not protons. This means that the presence of quark nuggets reduces the neutronto-proton ratio, thereby lowering the production of ${ }^{4} \mathrm{He}$. The helium-production is very sensitive to the total amount of nugget-area present, and in order not to ruin the concordance with observations, one finds [60 that only nuggets with $A>$ $A_{\mathrm{BBN}} \approx 10^{23} \Omega_{\text {nug }}^{3} h^{6} f_{n}^{3}$ are allowed during nucleosynthesis. Here $\Omega_{\text {nug }}$ is the present- 
day nugget contribution to the cosmic density (in units of the critical density), $h$ is the Hubble parameter in units of $100 \mathrm{~km} \mathrm{sec}^{-1} \mathrm{Mpc}^{-1}$, and $f_{n} \leq 1$ is the penetrability of the nugget surface. Slightly stronger limits can be obtained from inclusion of all light nuclei instead of ${ }^{4} \mathrm{He}$ only [61]. (Ref. 62 found good correspondence with nucleosynthesis for a nugget-dominated, $\Omega=1$ Universe if $A \approx 10^{17}$, but as shown in [46], this was due to an erroneous emission rate for nucleons.)

The nucleosynthesis calculations leading to $A_{\mathrm{BBN}}$ neglected inhomogeneities in the nucleon distribution, and all nuggets were assumed to have the same baryon number. However, the formation of ${ }^{4} \mathrm{He}$ is an on-off process over a limited range of $A$, so the detailed behavior of the inhomogeneities may not be so important.

Note that SQM, in spite of it carrying baryon number, does not contribute to the usual nucleosynthesis limit on $\Omega_{\text {baryon }}$. The SQM baryon number is "hidden" in quark nuggets long before Big Bang nucleosynthesis begins, and the nuggets only influence nucleosynthesis if they have a big total surface area, as described above.

Evaporating nuggets would lead to strongly inhomogeneous nucleosynthesis with enhanced heavy-element formation. This aspect has recently been studied in 63].

\subsection{Quark nuggets as dark matter}

Witten [2] argued that quark nuggets might be a natural explanation of the cosmological dark matter problem, in principle allowing a calculation of the relative amount of dark matter and ordinary baryons. In view of the evaporation discussed above, this idea now seems less likely, but is certainly not ruled out for $A>10^{30}$. Massive quark nuggets decouple from thermal equilibrium with the radiation bath very early in the history of the Universe, quickly slow down, and behave as cold dark matter in the context of galaxy formation.

Of course it should again be noted, that all of the interesting cosmological consequences of the quark-hadron phase transition require the transition to be first order, in agreement with recent lattice QCD calculations.

\section{$5 \quad$ SQM in neutron stars; strange stars}

It has been known for many years, that neutron stars may in fact be "hybrid stars" consisting of "ordinary" nuclear matter in the outer parts and quark matter in the central regions. This will be the case if SQM is metastable at zero pressure, being stabilized relative to hadronic matter by the high pressure within a neutron star [64, 65, 66].

If SQM is absolutely stable at zero pressure, an even more intriguing possibility opens up, namely the existence of "strange stars" [2, 67, 68, 69] consisting completely of SQM (perhaps apart from a minor crust to be discussed below). Such strange stars behave quite differently from neutron stars due to the unusual equation of state. For 
massless quarks the total energy density is given by $\rho=\rho_{q}+B$, and the total pressure by $P=P_{q}-B$, where $\rho_{q}$ is the energy density of quarks, and the pressure of the quarks is $P_{q}=\rho_{q} / 3$, since massless quarks are relativistic. The equation of state is thus given by

$$
P=\frac{1}{3}(\rho-4 B)
$$

The exact equation of state taking into account $m_{s} \neq 0$ is very similar 68 since $s$-quarks are relativistic for low $m_{s}$ and not present for high $m_{s}$. (I here assume that $\alpha_{s}=0$, but recall from Section 2.3, that a non-zero $\alpha_{s}$ effectively corresponds to a reduction of $B$ ).

The structure of a strange star is calculated from the Oppenheimer-Volkoff equation, describing the balance between gravity and pressure gradient, using the equation of state given above. The surface of the star corresponds to $P=0$, a condition fulfilled for $\rho=4 B$, which for typical values of $B$ is somewhat more than the density of ordinary nuclear matter! For stellar masses below $1 M_{\odot}\left(M_{\odot}\right.$ is the solar mass) this density is almost constant throughout the star, so to a good approximation total mass and radius are related by $M \propto R^{3}$, a relation in striking contrast to ordinary neutron stars, where $M \propto R^{-3}$. This means that low-mass neutron stars and strange stars have widely different radii, possibly allowing observational distinction. Unfortunately Nature prefers to form these compact objects with masses near $1.4 M_{\odot}$, according to stellar evolution models. For such a mass gravity rather than bag pressure plays the dominant stabilizing role, and there is no significant difference between neutron star and strange star radii. Also the maximum mass given by gravitational instability (the Chandrasekhar limit) is similar, of order $2 M_{\odot}$. In contrast to ordinary neutron stars, which are unstable for masses below $0.1 M_{\odot}$, strange stars have no minimum mass; the sequence continues smoothly to the domain of strangelets.

For the simple equation of state discussed above, the only natural energy scale in the problem is $B^{1 / 4}$. Thus there exists a homology transformation between strange star models for different values of $B$. In particular, the maximum mass of a strange star is given by

$$
M_{\max }=2.006 B_{145}^{-1 / 2} M_{\odot} .
$$

The corresponding minimal radius, maximal moment of inertia, maximal central density, surface density, and minimal rotation period (the so-called Kepler period corresponding to mass-shedding at the equator), are given by

$$
\begin{gathered}
R_{\min }=10.94 B_{145}^{-1 / 2} \mathrm{~km}, \\
I_{\max }=2.256 \times 10^{45} B_{145}^{-3 / 2} \mathrm{~g} \mathrm{~cm}^{2}, \\
\rho_{\max }=1.97 \times 10^{15} B_{145 \mathrm{~g} \mathrm{~cm}^{-3},} \\
\rho_{\text {surf }}=4.102 \times 10^{14} B_{145 \mathrm{~g} \mathrm{~cm}^{-3}},
\end{gathered}
$$




$$
P_{\text {min }}=0.66 B_{145}^{-1 / 2} \mathrm{~ms} .
$$

Bare strange stars (strange stars with quark matter all the way to the surface) have quite unusual properties. The density abruptly jumps from 0 to $\rho_{\text {surf }}$ (Eq. (80)), and the density is almost constant through the interior (except when the mass is close to $M_{\max }$ ). The plasma frequency of the star is huge, meaning that photons with energies below $20 \mathrm{MeV}$ are reflected from the surface, whereas the star itself can only emit photons with higher energies [68, 70]. Even more important, because of the strong interaction binding of the surface material, the star is not subject to the "Eddington limit", which for ordinary neutron stars limits the luminosity to be below $10^{38} \mathrm{erg} / \mathrm{s}$ (for higher luminosities the radiation pressure would exceed the gravitational attraction and expel the surface layers). As discussed below, this could lead to important "applications" of strange stars.

This approach may however be oversimplified because real strange stars may have surfaces more like ordinary neutron stars. In particular, a solid crust of ordinary material may form from accretion by the strange star after formation, or from material that was not converted during neutron star burning (see Section 5.5). Such a crust may be held up by the extreme, outward directed electrostatic potential of $10^{17}$ $10^{18} \mathrm{~V} / \mathrm{cm}$, created by the electron atmosphere with a thickness of a few hundred Fermi. This atmosphere merely expresses that the electrostatic binding of electrons is weaker than the strong binding of quarks; therefore the electron distribution does not end abruptly like that of quarks (the detailed structure was found from a ThomasFermi calculation by Alcock, Farhi and Olinto [68]; see also [71]).

The electrostatic potential can sustain a significant crust of ordinary neutron star material. The limit is given by the neutron drip density $\left(4 \times 10^{11} \mathrm{gcm}^{-3}\right)$, above which neutrons drip out of nuclei and would be swallowed by the quark phase. This crust may be decisive for interpretation of pulsar behavior (Section 5.2).

As emphasized by Glendenning, Kettner and Weber [72, 73], the existence of crusts not only changes the mass-radius relation for strange stars, but also opens a rich plethora of new stellar configurations. In particular, one may have a sequence of "strange dwarfs", much like white dwarfs except for an SQM core. At present there is no well-studied model for formation of such strange dwarfs.

Another possibility for formation of a (solid?) crust has been suggested in 74 . This mechanism relies on the existence of stable, low-baryon number strangelets (in this context sometimes denoted "quark-alphas" for the $A=6$ strangelet analog of a helium nucleus [75]) which could act as "nuclei" in the surface region. Whereas this possibility may seem less likely from the discussion in Section 3, it can not be entirely ruled out.

Finally, it is worth noticing that Glendenning [76] has argued that neutron stars may contain regions with mixed quark and hadron phases. (This possibility was missed in earlier studies due to an erroneous assumption of local rather than global charge neutrality). Depending on parameters the mixed phase region can occupy 
a significant fraction of the star, and may show unusual topologies (plate-like or cylinder-like structures, rather than just spherical quark bubbles embedded in hadrons or vice versa [77, 78, 79]).

Studies of strange stars have not been pursued to the degree of detail known for ordinary neutron stars, and it is premature to draw any detailed conclusions. However, in the following, I shall look at some of the properties expected and emphasize the possible observable differences between neutron stars and strange stars.

\section{$5.1 \quad$ Neutrino cooling}

A distinction between strange stars and neutron stars was for a long time believed to be a much more rapid cooling of SQM due to neutrino emitting weak interactions involving the quarks [68]. Thus a strange star was presumed to be much colder than a neutron star of similar age, a signature potentially observable from x-ray satellites. Only a few speculative mechanisms, such as the existence of kaon condensates might mimic the speed of quark matter neutrino cooling. Recently the story has been complicated considerably by the finding that ordinary neutron $\beta$-decay may be energetically allowed in nuclear matter [80], so that the cooling rate can be comparable to that of SQM. For this reason I shall not discuss the issue here, but refer the reader to an excellent review of neutron star cooling by Pethick [81, and a recent reinvestigation of strange star cooling by Schaab et al. [82].

\subsection{Pulsar glitches}

One important feature seems to distinguish strange stars from neutron stars in a manner with observable consequences, and that is the distribution of the moment of inertia inside the star. Ordinary neutron stars older than a few months have a crust made of a crystal lattice or an ordered inhomogeneous medium reaching from the surface down to regions with density $2 \times 10^{14} \mathrm{~g} \mathrm{~cm}^{-3}$. This crust contains about $1 \%$ of the total moment of inertia. Strange stars in contrast can only support a crust with density below the neutron drip density $\left(4.3 \times 10^{11} \mathrm{~g} \mathrm{~cm}^{-3}\right)$. This is because free neutrons would be absorbed and converted by the strange matter. Such a strange star crust contains at most a few times $10^{-5}$ of the total moment of inertia. This is an upper bound, since the strange star may have no crust at all, depending on its prior evolution. And recent studies of the mechanical balance between electric and gravitational forces on the crust indicate, that only densities up to perhaps $10^{11} \mathrm{~g} \mathrm{~cm}^{-3}$ may be achieved [83, 84].

As stressed by Alpar [85], and also pointed out by Haensel, Zdunik, and Schaeffer [67], and by Alcock, Farhi, and Olinto [68], this difference in the moment of inertia stored in the crust of neutron stars and strange stars seems to pose significant difficulties for explaining the glitch-phenomenon observed in radio pulsars 
with models based on strange stars. Glitches are observed as a sudden speedup in the rotation rate of pulsars. The fractional change in rotation rate $\Omega$ is $\Delta \Omega / \Omega \approx 10^{-6}-10^{-9}$, and the corresponding fractional change in the spin-down rate $\dot{\Omega}$ is of order $\Delta \dot{\Omega} / \dot{\Omega} \approx 10^{-2}-10^{-3}$. Regardless of the detailed model for the glitch phenomenon these jumps must involve the decoupling and recoupling of a component in the star containing a significant fraction, $I_{i} / I$, of the total moment of inertia; $f I_{i} / I=\Delta I / I \approx \Delta \Omega / \Omega \approx 10^{-6}-10^{-9}$ (Alpar actually argued that $f I_{i} / I \approx \Delta \dot{\Omega} / \dot{\Omega} \approx 10^{-2}-10^{-3}$, where $f$ is the fractional change in $I_{i}$, but this is not necessary [86]). This role is played by the inner crust of an ordinary neutron star, but the crust around a strange star is smaller; less than a few times $10^{-5} M_{\odot}$ with $I_{\text {crust }} / I$ around a few times $10^{-5}$ for ordinary neutron star masses of $1.4 M_{\odot}$ (higher for less massive stars). These numbers are based on models by Glendenning and Weber [86] assuming a maximum mass crust, i.e. a crust reaching neutron drip density at the base, so it seems fair to conclude, that strange stars in fact may have sufficiently massive crusts to account for glitches, but that parameters in that case are fairly tightly constrained.

Other possibilities for glitches in strange stars could involve a crust composed of strangelets (cf. the "quark-alpha" scenario in [74]), not to mention the possibility of a quark-hadron mixed phase [76, 77, 78, 79]. There is still a lack of any detailed model for how the magnetic field structure and other crucial aspects of a pulsar can be modeled for strange stars. Presumably a strange star cannot do the job without significant structure, such as a crust and/or superfluidity/superconductivity in certain regions. These issues have only been very superficially studied and need further consideration. The present lack of such models should not be used to dismiss the possibility of strange stars.

\subsection{Strange star oscillation and maximum rotation rate}

One of the most interesting differences between neutron stars and strange stars is related to the damping of instabilities.

First it should be noticed that a strange star is a very stable system. Strange stars may have radial oscillations with a fundamental period of $0.06-0.3 \mathrm{~ms}$ [87], but these are characterized by rapid damping in a matter of seconds [88, 89, 90, 91]. This is due to the extremely high viscosity of SQM.

The large viscosity also plays a role in setting the maximum rotation limit for strange pulsars (or hybrid stars with SQM cores). The ultimate rotation limit corresponds to mass-shedding from the equator of the star (this is called the Kepler limit and is of order $0.6 \mathrm{msec}$ for a strange star, Eq. (81); see Zdunik 92 for a review). But before reaching such rotation rates, the pulsars become unstable to non-radial deformations and are slowed down by emission of gravitational radiation. Shear and bulk viscosities tend to stabilize the star against these instabilities [89, 93], and the 
high value for the bulk viscosity may mean that strange pulsars in contrast to ordinary pulsars can reach submillisecond periods [91]. Thus the discovery of very fast pulsars may be an indication favoring the existence of strange stars.

And even more exciting, it has been shown over the last few months, that ordinary neutron stars when they are young and hot are subject to a new class of instabilities, called r-mode instabilities [94, 95, 96], which during their first year of existence slows the rotation rate to only a few per cent of the Kepler limit. Rotation periods faster than 10 msec are unlikely after that, until some pulsars at a much later age may be spun-up by angular momentum transfer in binary systems, and thereby explain the rapid old pulsars with periods down to $1.56 \mathrm{msec}$. In contrast, strange stars are not subject to these instabilities until they are thousands of years old, and even then only for periods faster than $2-3$ msec [97]. This seems to imply, that the most robust signature for the existence of strange stars (or neutron stars with a substantial fraction of high viscosity quark matter in the interior) is to search for young pulsars with rotation periods below, say, 5 msec (even stars with longer periods may candidate). These can not be ordinary neutron stars, whereas quark matter is the only substance known to have a bulk viscosity high enough to offer an explanation.

The bulk viscosity of strange quark matter depends on the rate of the non-leptonic interaction

$$
u+d \leftrightarrow s+u
$$

(The rate for this reaction has recently been calculated by Madsen [35], and Heiselberg [36]; earlier studies, including that of Ref. [33] are incorrect). This reaction changes the concentrations of down and strange quarks in response to the density changes involved in vibration or rotational instabilities, thereby causing dissipation. This dissipation is most efficient if the rate of reaction (82) is comparable to the frequency of the density change. If the weak rate is very small, the quark concentrations keep their original values in spite of a periodic density fluctuation, whereas a very high weak rate means that the matter immediately adjusts to follow the true equilibrium values reversibly. But in the intermediate range dissipation due to $P d V$-work is important.

The importance of dissipation due to Eq. (82) was first stressed by Wang and Lu [88] in the case of neutron stars with quark cores. These authors made a numerical study of the evolution of the vibrational energy of a neutron star with an $0.2 M_{\odot}$ quark core, governed by the energy dissipation due to Eq. (82). Sawyer [89 expressed the damping in terms of the bulk viscosity, a function of temperature and oscillation frequency, which he tabulated for a range of densities and strange quark masses. Sawyer's tabulation has later been used in studies of quark star vibration [90], and of the gravitational radiation reaction instability determining the maximum rotation rate of pulsars [93. The latter study concluded, that the bulk viscosity is large enough to be important for temperatures exceeding $0.01 \mathrm{MeV}$, but that it should be a few orders of magnitude larger to generally dominate the stability properties. 
However, as has been pointed out in [91], the bulk viscosities calculated in [89] depend on the assumption, that the rate of Eq. (82) can be expanded to first order in $\delta \mu=\mu_{s}-\mu_{d}$, where $\mu_{i} \approx 300 \mathrm{MeV}$ are the quark chemical potentials. This assumption is not correct at low temperatures $(2 \pi T \ll \delta \mu)$, where the dominating term in the rate is proportional to $\delta \mu^{3}$. Furthermore, the rate in [89] is too small by an overall factor of 3 , and a discrepancy of 2-3 orders of magnitude, perhaps due to unit conversions, appears as well. Taken together, these effects lead to an upward correction of the bulk viscosity by several orders of magnitude, and thereby increases the importance for the astrophysical applications. The non-linearity of the rate also means, that the bulk viscosity is no longer independent of the amplitude of the density variations. The resulting bulk viscosity is (in cgs-units, with $m_{s}, T$, and $\mu_{d} \approx 235 \mathrm{MeV}\left(\rho / \rho_{\text {nuc }}\right)^{1 / 3}$ in $\mathrm{MeV}$, and the oscillation frequency $\omega$ in $\left.\mathrm{s}^{-1}\right)$

$$
\zeta \approx 3.09 \times 10^{28} m_{s}^{4} \omega^{-2}\left(\frac{\rho}{\rho_{\text {nuc }}}\right)\left[\frac{3}{4}\left(\frac{m_{s}^{2}}{3 \mu_{d}} \frac{\Delta v}{v_{0}}\right)^{2}+4 \pi^{2} T^{2}\right] \mathrm{g} \mathrm{cm}^{-1} \mathrm{~s}^{-1} .
$$

For typical values $\left(m_{s}=100 \mathrm{MeV}, \mu_{d}=300 \mathrm{MeV}, \omega=2 \times 10^{4} \mathrm{~s}^{-1}\right)$ this is $\zeta \approx$ $1.6 \times 10^{28}\left[93\left(\Delta v / v_{0}\right)^{2}+39 T^{2}\right] \mathrm{g} \mathrm{cm}^{-1} \mathrm{~s}^{-1}$, where $\Delta v / v_{0}$ is the perturbation amplitude.

For a star of constant density (an excellent approximation for a strange star, except very close to the gravitational instability limit) Sawyer [89] estimated the damping time as

$$
\tau_{D} \approx 1.5 \times 10^{25} \zeta^{-1} \mathrm{~s}
$$

Thus, even at very low temperatures, high amplitude oscillations are damped in fractions of a second, and those of low amplitude in a matter of minutes, if one takes into account, that the temperature of the star increases due to the heat released by viscous dissipation, which can speed up the damping of vibrations.

The discussion above was based on rather crude estimates [91]. A detailed, general relativistic, numerical treatment along the lines of Cutler et al. 90 is clearly needed.

As mentioned previously, viscosity also plays an important role in setting the maximum rotation rate of pulsars. Gravitational radiation reaction instabilities (as opposed to "Keplerian mass-shedding") is supposed to set the ultimate rotation rate limit, but the larger the damping by shear and bulk viscosity is, the closer the rate can get to the Keplerian limit given in Eq. (81).

The shear viscosity of SQM due to quark scattering has recently been recalculated by Heiselberg and Pethick [98. Their results for $T \ll \mu$ can be written as

$$
\eta \approx 4.0 \times 10^{15}\left(\frac{0.1}{\alpha_{S}}\right)^{5 / 3}\left(\frac{\rho}{\rho_{\text {nuc }}}\right)^{14 / 9} T^{-5 / 3} \mathrm{gcm}^{-1} \mathrm{~s}^{-1} .
$$

Investigations by Colpi and Miller [93] based on the older viscosities in [89, 99] indicated, that the minimal rotation period of strange stars might be set by the gravitational radiation reaction instability of $m=2$ or $m=3$ modes at or just below 
1 millisecond. With the new, much larger, viscosities, the non-axisymmetric instabilities will be suppressed, and it is not unreasonable to expect, that the maximum rotation frequency of strange stars will be close to the Keplerian limit. Detailed numerical calculations like those in Colpi and Miller [93], including the new viscosities and effects of dissipative heating, are required to settle the issue, but they are complicated by the non-linear behavior of the new bulk viscosity.

Whether or not the ultimate rotation period of strange stars can be significantly smaller than for neutron stars is of importance for old pulsars spun-up by accretion. But perhaps the most clear-cut signature for the existence of strange stars would be the (almost) lack of sensitivity to r-mode instabilities, which as mentioned earlier allows young strange stars to rotate much faster than young neutron stars [97].

\subsection{Gamma-ray bursters}

Strange stars because of their high surface density, strong binding (making it possible to circumvent the Eddington limit), and special emission properties have been suggested as explanations for some of the more mysterious cosmic events, namely $\gamma$-ray bursters. These are bursts of $\gamma$-rays of a few seconds duration, coming from unidentified sources which are presumably at extragalactic distances.

No consensus exists concerning the nature of these bursts, but Alcock, Farhi and Olinto [100] suggested a detailed model for the most prominent of the bursters, the one on 5 March 1979. Their model is based on an impact of a $10^{-8} M_{\odot}$ lump of SQM on a rotating strange star, and the authors are able to explain most of the observations concerning energetics and time-scales under the assumption that the burster is located in a supernova remnant in the Large Magellanic Cloud, as position measurements seem to indicate. An alternative model for this source and for soft $\gamma$-repeaters in the framework of strange stars with "quark-alpha" surface properties was suggested in 101]. Other strange star models for soft $\gamma$-repeaters and $x$-ray bursters include 102, 103].

$\gamma$-ray bursters at truly cosmological distances could be due to collisions of two strange stars in binary systems [104], each collision releasing $10^{50} \mathrm{ergs}$ in the form of gamma rays over a time-scale of $0.2 \mathrm{~s}$.

There are, however, literally hundreds of different models for $\gamma$-bursts, and in spite of improved observational data the interpretation is at present unclear.

A recent identification of the $x$-ray source Her X-1 as a strange star [105] was unfortunately based on incorrect use of bag model parameters [106].

\subsection{Formation of strange stars}

If strange quark matter is stable, strange stars may be formed during supernovaexplosions, and neutron stars can be converted to strange stars by a number of 
different mechanisms, such as pressure-induced transformation to uds-quark matter via ud-quark matter, sparking by high-energy neutrinos, or triggering due to the intrusion of a quark nugget. These and other possibilities were described by Alcock, Farhi, and Olinto [68].

As soon as a lump of strange matter comes in contact with free neutrons it starts converting them into strange matter. The burning of a neutron star into a strange star was discussed by Baym et al. [107] and Olinto [108], and it was shown that the star would be converted on a rather small time-scale set by quark diffusion and flavor-changing weak interactions. (The huge difference in the speed of the conversion front found in these papers is partly due to the omission of a factor $c^{1 / 2}$, where $c$ is the speed of light, in equation (6) of Baym et al.) Later studies [109, 110 found burning times in the range of $1-10^{3}$ seconds under various parameter assumptions (see also Olinto 111 for a review). For the fastest burning times, the energy liberated may be important for the supernova mechanism and supernova neutrino bursts. Horvath and Benvenuto [112 have questioned the stability of "slow" neutron combustion and suggested that the conversion takes place much faster as a detonation. So far, the investigations of neutron star burning have been rather crude, neglecting many aspects of transport theory, heat conduction etc. A detailed study of this phenomenon would be interesting.

Perhaps the most likely mechanisms for initiating the formation of a strange star involves either a seed of SQM in the star (see Section [6), or thermal formation of quark matter bubbles. Thermal triggering of neutron star transformation may be understood qualitatively in terms of simple boiling theory. Before considering a more realistic equation of state it is instructive to study the boiling of a pure neutron gas into quarks. The quark bubbles formed consist of $u$ and $d$ quarks in the ratio 1:2; only later weak interactions may change the composition to an energetically more favorable state. Thus quark chemical potentials are related by $\mu_{d}=2^{1 / 3} \mu_{u}$, and $\mu_{n}=\mu_{u}+2 \mu_{d}=\left(1+2^{4 / 3}\right) \mu_{u}$, assuming chemical equilibrium across the phase boundary.

The free energy involved in formation of a spherical quark bubble of radius $R$ and volume $V$ is given by

$$
F=-\Delta P V+8 \pi \gamma R
$$

where

$$
\Delta P=P_{u d}-P_{n}=\frac{\mu_{u}^{4}+\mu_{d}^{4}}{4 \pi^{2}}-B-P_{n}
$$

and the curvature energy coefficient

$$
\gamma=\frac{\mu_{u}^{2}+\mu_{d}^{2}}{8 \pi^{2}}
$$

The free energy has a maximum at the critical radius

$$
r_{c}=(2 \gamma / \Delta P)^{1 / 2}
$$


and the corresponding free energy

$$
W_{c} \equiv F\left(r_{c}\right)=16 \pi \gamma r_{c} / 3
$$

is the work required to form a bubble of this radius which is the smallest bubble capable of growing. It is a standard assumption in the theory of bubble nucleation in first order phase transitions that bubbles form at this particular radius at a rate given byt

$$
\mathcal{R} \approx T^{4} \exp \left(-W_{c} / T\right)
$$

The simplest possible equation of state for the neutron gas is that of a zero temperature, nonrelativistic degenerate Fermi-gas, where

$$
P_{n}=\frac{\left(\mu_{n}^{2}-m_{n}^{2}\right)^{5 / 2}}{15 \pi^{2} m_{n}}
$$

and the baryon density

$$
n_{B}=\frac{\left(\mu_{n}^{2}-m_{n}^{2}\right)^{3 / 2}}{3 \pi^{2}}
$$

A necessary condition for boiling is that $\Delta P>0$. This leads to an upper limit on the bag constant, $B_{\max }$, from Eq. (87) as illustrated in Fig. 6 (from [113 - the corresponding limit for the Bethe-Johnson equation of state is shown for comparison; it is seen to be very similar). This was also used as a criterion for neutron star stability by Krivoruchenko and Martemyanov [114].

Also shown in Fig. 6 is the limit on the bag constant below which bubble nucleation takes place at rates exceeding $1 \mathrm{~km}^{-3} \mathrm{Gyr}^{-1}$ and $1 \mathrm{~cm}^{-3} \mathrm{~s}^{-1}$, respectively, for temperatures of $1,2,3$ and $10 \mathrm{MeV}$ ( $B_{\max }$ can be considered as the limit for infinite temperature). One notes that the possibility of bubble nucleation is fairly insensitive to the temperature as soon as $T$ exceeds a few $\mathrm{MeV}$, whereas thermally induced bubble nucleation is impossible for $T<2 \mathrm{MeV}$ (recall from Section 2.2 that the stability of ordinary nuclei against decay into quark matter requires that $\left.B>(145 \mathrm{MeV})^{4}\right)$. This confirms an estimate in [115] (see also [116, 117]). The range of bag constants for which a hot neutron star may transform into quark matter is thus roughly $145 \mathrm{MeV}<B^{1 / 4}<155 \mathrm{MeV}$.

Results for a more realistic mean field equation of state are also shown in the Figure (see [113] for further details). While the detailed numbers change, the overall conclusion does not. Quark matter bubbles may nucleate (possibly followed by burning of the star into SQM) in neutron stars/supernovae if the bag constant is low, and if the temperature exceeds a few $\mathrm{MeV}$ (thus the process is most likely during the supernova explosion itself). Should thermal nucleation not take place, one of the other mechanisms mentioned above must be relied on. Apart from seed-induced burning, all of these are likely to be much less efficient than thermal nucleation.

\footnotetext{
${ }^{2}$ The prefactor may differ from $T^{4}$, but this is of minor practical importance due to the dominant exponential.
} 

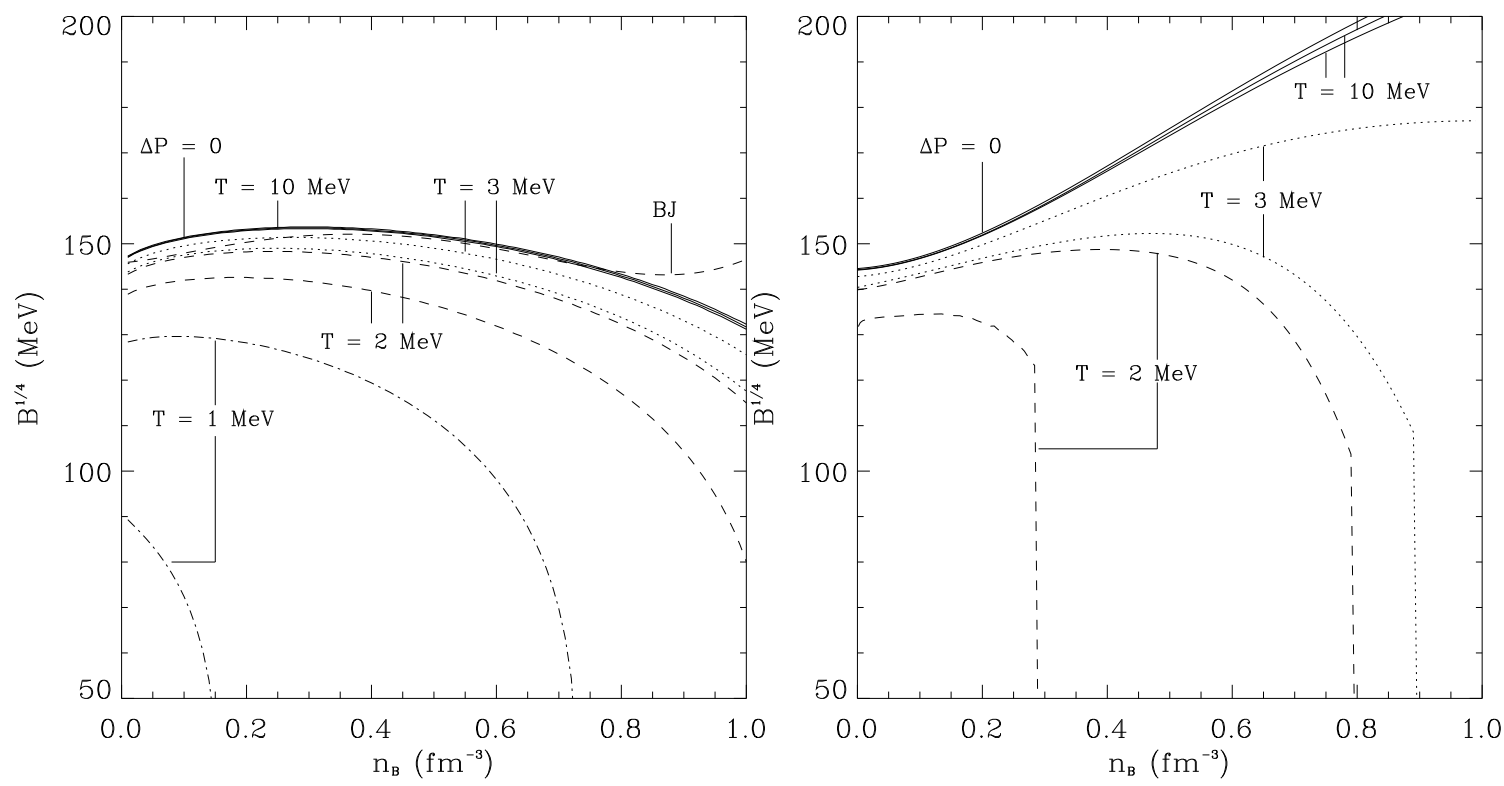

Figure 6: Upper limits on the bag constant allowing thermal nucleation of quark matter bubbles in neutron stars as a function of baryon number density in the hadron phase. To the left are shown results for the simple neutron gas model discussed in the text. To the right for a more realistic mean field approximation. See text for further explanations.

\section{$6 \quad \mathrm{SQM}$ in cosmic rays}

De Rújula and Glashow [118] argued that unusual meteor-events, earth-quakes, etched tracks in old mica, in meteorites and in cosmic-ray detectors might be used for observation of quark nuggets hitting the Earth or its atmosphere. In particular they were interested in the possibility of detecting a galactic dark matter halo of nuggets, where typical velocities would be a few hundred kilometers per second, given by the depth of the gravitational potential. Even if nuggets only survived from the Big Bang in small numbers, or were spread in our galaxy by secondary processes such as strange star collisions, there could be a potentially observable flux of nuggets hitting the Earth. The only data actually investigated in their paper came from a negative search for tracks in ancient mica, and corresponded to a lower nugget flux limit of $8 \times 10^{-19} \mathrm{~cm}^{-2} \mathrm{~s}^{-1} \mathrm{sr}^{-1}$, for nuggets with $A>1.4 \times 10^{14}$ (smaller nuggets would be trapped in layers above the mica samples studied). This can also be expressed as an excluded range of $1.4 \times 10^{14}<A<8 \times 10^{23} \rho_{24} v_{250}$, where $v \equiv 250 \mathrm{~km} \mathrm{~s}^{-1} v_{250}$ and $\rho \equiv 10^{-24} \mathrm{~g} \mathrm{~cm}^{-3} \rho_{24}$ are the typical speeds and mass density of nuggets in the galactic halo. The speed is given by the depth of the gravitational potential of our galaxy, whereas $\rho_{24} \approx 1$ corresponds to the density of dark matter. In these units the number 
of nuggets hitting the Earth per $\mathrm{cm}^{2}$ per second per steradian is $6.0 \times 10^{5} A^{-1} \rho_{24} v_{250}$.

Later investigations have improved these flux limits somewhat. These Earthbased flux-limits [119, 120] are shown in Figure 0. It is seen that quark nuggets with $3 \times 10^{7}<A<5 \times 10^{25}$ seem incapable of explaining the dark halo around our galaxy, but a low flux either left over from the Big Bang or arising from collision of strange stars cannot be ruled out. If the strange matter hypothesis is valid, one should indeed expect a significant background flux from stellar collisions, since several pulsars are members of binary systems, where the two components are ultimately going to collide. If such collisions spread as little as $0.1 M_{\odot}$ of non-relativistic strangelets with baryon number $A$, a single collision will lead to a flux of $10^{-6} A^{-1} v_{250} \mathrm{~cm}^{-2} \mathrm{~s}^{-1} \mathrm{sterad}^{-1}$, assuming strangelets to be spread homogeneously in a halo of radius $10 \mathrm{kpc}$.

Such a flux-level is below the sensitivity of present experiments, but Madsen [121] suggested that neutron stars and their stellar "parents" may be used as alternative large surface area, long integration time detectors. The reason is simple. The presence of a single quark nugget in the interior of a neutron star is sufficient to initiate a transformation of the star into a strange star [2, 68, 107]. The time-scale for the transformation is short, between seconds and minutes [107, 108, 111, 109, 110], so observed pulsars would have been converted long ago, if their stellar progenitors ever captured a quark nugget, or if the neutron stars themselves absorbed one after formation.

The rate at which quark nuggets hit the surface of a star depends on the phase space distribution of nuggets relative to the star. For an infinite bath of positive energy nuggets with an isotropic, monoenergetic distribution function, the number accretion rate is given by

$$
F=1.39 \times 10^{30} \mathrm{~s}^{-1} A^{-1} M R \rho_{24} v_{250}^{-1}\left[1+0.164 v_{250}^{2} R M^{-1}\right],
$$

where $M$ and $R$ denote the stellar mass and radius in solar units. For the Sun the second term in parenthesis (the geometrical term) contributes only slightly to the accretion rate, and the contribution is even less important for more massive stars and for compact objects like white dwarfs and neutron stars (in contrast, the geometrical term dominates for accretion onto the Earth). In the following I therefore only take the first term (gravitational) into account.

To convert a neutron star into strange matter a quark nugget should not only hit a supernova progenitor but also be caught in the core. Similarly, nuggets hitting a neutron star after its creation have to penetrate the outer layers and reach the neutron drip region. These issues were discussed in 121.

A main sequence star is capable of capturing quark nuggets with baryon numbers below $A_{\text {STOP, }}$ where

$$
A_{\mathrm{STOP}}=5.0 \times 10^{31} M^{-1.8} .
$$

This works for non-relativistic nuggets, which are basically braked by inertia, i. e. they are slowed down by electrostatic scatterings after plowing through a column of 
mass similar to their own, and afterwards settle in the stellar core. In particular it is valid for nuggets moving with virial speed in our galactic halo. Relativistic nuggets, like those reported in some cosmic ray observations, may be destroyed after collisions with nuclei in the stellar atmospheres, and so the limit can not be used immediately, but it is worth noticing, that even a tiny fraction of a nugget surviving such an event and settling in the star is sufficient to convert the neutron star to a strange star.

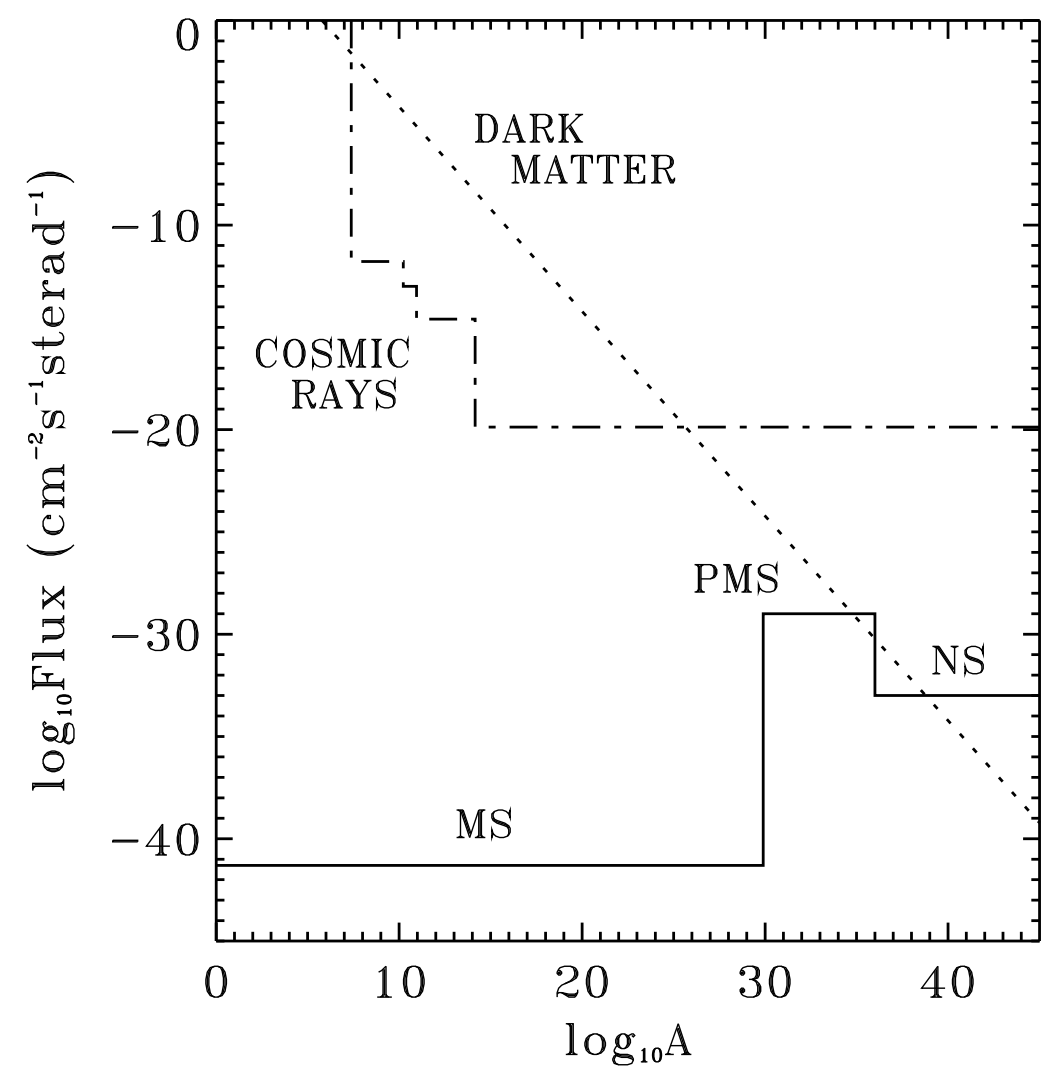

Figure 7: Astrophysical flux-limits [121] compared to the flux expected for a galactic halo of nuggets being the dark matter [118], and to the experimental results for cosmic rays [119, [120]. The three horizontal parts of the solid curve correspond to capture in main sequence supernova progenitors, post main sequence stars, and neutron stars younger than the Vela pulsar ( $10^{4}$ years).

For nuggets with $A<A_{\mathrm{STOP}}$ the sensitivity of main sequence stars as detectors is remarkable, as it is given by the limit of one nugget hitting the surface of the supernova progenitor in its main sequence lifetime! Converted into a flux, $\mathcal{F}$, of 
nuggets hitting the Earth per $\mathrm{cm}^{2}$ per sec per steradian, it corresponds to

$$
\mathcal{F}=4 \times 10^{-42} M^{0.1} v_{250}^{2}
$$

As can be seen from Figure 7, this is a factor of $10^{20}-10^{40}$ more sensitive than ordinary experiments!

If it is possible to prove that some neutron stars are indeed neutron stars rather than strange stars, the sensitivity of the astrophysical detectors rules out quark nuggets as being the dark matter for baryon numbers in the range $A<10^{34-38}$. And it questions the whole idea of stable strange quark matter, since it seems impossible to avoid polluting the interstellar medium with nuggets from strange star collisions or supernova explosions at fluxes many orders of magnitude above the limit measurable in this way.

If on the other hand SQM is stable, then all neutron stars are likely to be strange stars, again because some pollution can not be avoided.

The Sun would in this way accrete $3.7 \times 10^{-20} \rho_{24} v_{250}^{-1} M_{\odot} /$ year, or a total of $10^{-10} \rho_{24} v_{250}^{-1} M_{\odot}$ in its total lifetime on the main sequence. Very low-mass nuggets collected near the solar center in this manner might have an impact on the energy production [122], but the effect is negligible unless the electrostatic barrier at the nugget surface is much smaller than expected, or unless very special circumstances allow nuggets to catalyze nuclear reactions [21].

The Sun will develop into a white dwarf in about $6 \times 10^{9}$ years. As just mentioned, the Sun would accrete a core of $10^{-10} \rho_{24} v_{250}^{-1} M_{\odot}$ in its total lifetime on the main sequence. Such accretion is too small to lead to a strange dwarf distinguishable from an ordinary white dwarf as suggested in [72, 73]. However, higher concentrations could occur if quark nuggets were somehow mixed into the gas cloud from which the star originally formed. Whether this is likely to happen depends strongly on assumptions regarding the velocity distribution of the nuggets formed, and the possibility of interactions with the gas 123 .

Most of the discussion above dealt with halo nuggets moving at non-relativistic velocities. Relativistic nuggets are not as easily detected using neutron stars, since they may be destroyed in collisions with nuclei in the star. On the other hand two relativistic candidate events with charge $Z=14$ and mass $A \approx 370$ were found in a balloon experiment by Saito et al. [32]. This corresponds to a rather high flux, and it is not quite clear how to produce such nuggets, though spallation of larger nuggets originating from strange star collisions may be involved [124].

Quark nuggets have also been suggested as candidates for the Centauro cosmic-ray events [2, 125, 126]. Centauro primaries may have a flux as high as $10^{-14} \mathrm{~cm}^{-2} \mathrm{~s}^{-1}$ and $A \approx 10^{3}$. Since Centauro primaries move at relativistic speeds they are destroyed by inelastic collisions when hitting a star, so the flux-limits given above cannot directly be used to rule out quark nuggets as Centauro primaries. However the mechanism producing the primaries must be tuned so that it only produces relativistic quark 
nuggets in order not to conflict with the flux-limits in Figure 7 for non-relativistic nuggets.

\section{Conclusion and outlook}

The possible stability of strange quark matter is a fundamentally exciting idea. Should it turn out to be true, many textbooks in nuclear, particle and astrophysics will need revisions, but our daily lives will not be changed dramatically, apart from possible technological applications such as energy production and disposal of radioactive waste [14, 127]. There are two main reasons why stability of SQM is possible without drastic consequences. The first reason is that stability requires a certain minimum strangeness content, so ordinary nuclei do not decay into strangelets. The second reason is the positive electrostatic potential of the quark phase in a strangelet, which means that you could walk around with a lump of SQM in your pocket without being swallowed.

While heavy-ion collisions is the way to look for small (meta)stable strangelets, astrophysics gives a possibility for testing larger (and therefore more stable) SQMsystems. Direct cosmic ray searches is an obvious way to look for intermediate baryon numbers in the form of relativistic or non-relativistic lumps produced in strange star collisions, and for leftovers from the Big Bang. The latter can only exist for very high baryon numbers (cf. Section 4), whereas a galactic background of the former seems unavoidable if the strange matter hypothesis is correct.

Strange stars may be the most promising place to look for SQM, but as explained in Section 5 it is actually hard to find clear-cut ways of distinguishing strange stars from neutron stars, unless one finds an object of very low mass. Pulsar rotation properties at present seem to provide the best clue, in particular after the finding that young strange stars in contrast to neutron stars are not braked by gravitational wave emission due to r-mode instabilities.

If SQM is only metastable, heavy-ion physicists may still have a chance of finding it; the cosmological quark-hadron phase transition may still lead to inhomogeneities of importance for Big Bang nucleosynthesis (without quark nuggets left over); and neutron stars may still have strange matter cores.

In any case the confirmation or disproof of the existence of (meta)stable strange quark matter via experiments and astrophysical tests makes it possible to limit strong interaction parameters that are otherwise difficult to probe. This in itself is a good reason for continued studies of the physics and astrophysics of strange quark matter. 


\section{Acknowledgments}

This work was supported in part by the Theoretical Astrophysics Center under the Danish National Research Foundation. I take this opportunity to thank the "strangers" among my present and former PhD-students, Michael Olesen, Dan Jensen, Michael Christiansen, and Gregers Neergaard for collaboration and many enlightening discussions.

\section{References}

[1] A. R. Bodmer, Phys. Rev. D 4, 1601 (1971).

[2] E. Witten, Phys. Rev. D 30, 272 (1984).

[3] Strange Quark Matter in Physics and Astrophysics, edited by J. Madsen and P. Haensel (Nucl. Phys. B (Proc. Suppl.), 24B, 1991).

[4] C. Alcock and A. Olinto, Annu. Rev. Nucl. Part. Sci. 38, 161 (1988).

[5] B. S. Kumar, in Physics and Astrophysics of Quark-Gluon Plasma, edited by B. Sinha, Y. P. Viyogi, and S. Raha (World Scientific, Singapore, 1994), pp. 63-74.

[6] B. S. Kumar, in Strangeness and Quark Matter, edited by G. Vassiliadis, A. D. Panagiotou, S. Kumar, and J. Madsen (World Scientific, Singapore, 1995), pp. 318-332.

[7] B. S. Kumar, Nucl. Phys. A 590, 29c (1995).

[8] J. Madsen, in Physics and Astrophysics of Quark-Gluon Plasma, edited by B. Sinha, Y. P. Viyogi, and S. Raha (World Scientific, Singapore, 1994), pp. 186205.

[9] J. Madsen, in Strangeness and Quark Matter, edited by G. Vassiliadis, A. D. Panagiotou, S. Kumar, and J. Madsen (World Scientific, Singapore, 1995), pp. 191-205.

[10] J. Madsen, in Strangeness in Hadronic Matter, Vol. 340 of AIP Conf. Proc., edited by J. Rafelski (AIP, New York, 1995), pp. 32-45.

[11] P. Papazoglou et al., in Strangeness and Quark Matter, edited by G. Vassiliadis, A. D. Panagiotou, S. Kumar, and J. Madsen (World Scientific, Singapore, 1995), pp. 206-219.

[12] C. Greiner and J. Schaffner-Bielich, preprint nucl-th/9801062. 
[13] S. Kabana for the NA52 collaboration, J. Phys. G 23, 2135 (1997).

[14] G. L. Shaw, M. Shin, R. H. Dalitz, and M. Desai, Nature 337, 436 (1989).

[15] S. L. Shapiro and S. A. Teukolsky, Black Holes, White Dwarfs, and Neutron Stars (John Wiley \& Sons, New York, 1983).

[16] E. Farhi and R. L. Jaffe, Phys. Rev. D 30, 2379 (1984).

[17] A. Chodos et al., Phys. Rev. D 9, 3471 (1974).

[18] T. A. DeGrand, R. L. Jaffe, K. Johnson, and J. Kiskis, Phys. Rev. D 12, 2060 (1975).

[19] D. Vasak, W. Greiner, and L. Neise, Phys. Rev. C 34, 1307 (1986).

[20] C. Greiner, D.-H. Rischke, H. Stöcker, and P. Koch, Phys. Rev. D 38, 2797 (1988).

[21] K. Takahashi and R. N. Boyd, Astrophys. J. 327, 1009 (1988).

[22] E. P. Gilson and R. L. Jaffe, Phys. Rev. Lett. 71, 332 (1993).

[23] J. Madsen, Phys. Rev. D 50, 3328 (1994).

[24] J. Schaffner-Bielich, C. Greiner, A. Diener, and H. Stöcker, Phys. Rev. C 55, 3038 (1997).

[25] M. S. Desai, H. J. Crawford, and G. L. Shaw, Phys. Rev. D 47, 2063 (1993).

[26] H. J. Crawford, M. S. Desai, and G. L. Shaw, Phys. Rev. D 48, 4474 (1993).

[27] M. S. Berger and R. L. Jaffe, Phys. Rev. C 35, 213 (1987).

[28] J. Madsen, Phys. Rev. Lett. 70, 391 (1993).

[29] J. Madsen, Phys. Rev. D 47, 5156 (1993).

[30] H. Heiselberg, Phys. Rev. D 48, 1418 (1993).

[31] R. Balian and C. Bloch, Ann. Phys. 60, 401 (1970).

[32] T. Saito, Y. Hatano, Y. Fukada, and H. Oda, Phys. Rev. Lett. 65, 2094 (1990).

[33] H. Heiselberg, J. Madsen, and K. Riisager, Phys. Scr. 34, 556 (1986).

[34] P. Koch, in Strange Quark Matter in Physics and Astrophysics, edited by J. Madsen and P. Haensel (Nucl. Phys. B (Proc. Suppl.), 24B, 1991), pp. 255-259. 
[35] J. Madsen, Phys. Rev. D 47, 325 (1993).

[36] H. Heiselberg, Phys. Scr. 46, 485 (1992).

[37] D. M. Jensen and J. Madsen, Phys. Rev. D (Rapid Communication) 53, R4719 (1996).

[38] Y. B. He, C. S. Gao, X. Q. Li, and W. Q. Chao, Phys. Rev. C 53, 1903 (1996).

[39] M. G. Mustafa and A. Ansari, Phys. Rev. D 53, 5136 (1996); Erratum ibid 54, 4694 (1996).

[40] M. G. Mustafa and A. Ansari, Phys. Rev. C 55, 2005 (1997).

[41] J. H. Applegate and C. J. Hogan, Phys. Rev. D 31, 3037 (1985).

[42] H. Kurki-Suonio, Phys. Rev. D 37, 2104 (1988).

[43] K. Sumiyoshi, T. Kajino, C. R. Alcock, and G. J. Mathews, Phys. Rev. D 42, 3963 (1990).

[44] K. Jedamzik and G. M. Fuller, Astrophys. J. 423, 33 (1994).

[45] C. Alcock and E. Farhi, Phys. Rev. D 32, 1273 (1985).

[46] J. Madsen, H. Heiselberg, and K. Riisager, Phys. Rev. D 34, 2947 (1986).

[47] K. Sumiyoshi and T. Kajino, in Strange Quark Matter in Physics and Astrophysics, edited by J. Madsen and P. Haensel (Nucl. Phys. B (Proc. Suppl.), 24B, 1991), pp. 80-83.

[48] P. Bhattacharjee, J. Alam, B. Sinha, and S. Raha, Phys. Rev. D 48, 4630 (1993).

[49] C. Alcock and A. Olinto, Phys. Rev. D 39, 1233 (1989).

[50] J. Madsen and M. L. Olesen, Phys. Rev. D 43, 1069 (1991); Erratum ibid 44, 566 (1991).

[51] M. L. Olesen and J. Madsen, Phys. Rev. D 47, 2313 (1993).

[52] H.-C. Liu and G. L. Shaw, Phys. Rev. D 30, 1137 (1984).

[53] C. Greiner, P. Koch, and H. Stöcker, Phys. Rev. Lett. 58, 1825 (1987).

[54] C. Greiner and H. Stöcker, Phys. Rev. D 44, 3517 (1991). 
[55] H. W. Barz, B. L. Friman, J. Knoll, and H. Schulz, in Strange Quark Matter in Physics and Astrophysics, edited by J. Madsen and P. Haensel (Nucl. Phys. B (Proc. Suppl.), 24B, 1991), pp. 211-220.

[56] H. J. Crawford, M. S. Desai, and G. L. Shaw, Phys. Rev. D 45, 857 (1992).

[57] C. Spieles et al., Phys. Rev. Lett. 76, 1776 (1996).

[58] C. H. Lee and H. K. Lee, Phys. Rev. D 44, 398 (1991).

[59] S. J. Cho, K. S. Lee, and U. Heinz, Phys. Rev. D 50, 4771 (1994).

[60] J. Madsen and K. Riisager, Phys. Lett. 158B, 208 (1985).

[61] M. L. Olesen, Master Thesis, University of Aarhus, 1990.

[62] R. Schaeffer, P. Delbourgo-Salvador, and J. Audouze, Nature 317, 407 (1985).

[63] K. Jedamzik, G. M. Fuller, and G. J. Mathews, Astrophys. J. 422, 423 (1994).

[64] G. Baym and S. A. Chin, Nucl. Phys. A 262, 527 (1976).

[65] G. Chapline and M. Nauenberg, Nature 264, 23 (1976).

[66] B. Freedman and L. McLerran, Phys. Rev. D 17, 1109 (1978).

[67] P. Haensel, J. L. Zdunik, and R. Schaeffer, Astron. Astrophys. 160, 121 (1986).

[68] C. Alcock, E. Farhi, and A. Olinto, Astrophys. J. 310, 261 (1986).

[69] C. Alcock, in Strange Quark Matter in Physics and Astrophysics, edited by J. Madsen and P. Haensel (Nucl. Phys. B (Proc. Suppl.), 24B, 1991), pp. 93-102.

[70] T. Chmaj, P. Haensel, and W. Słomiński, in Strange Quark Matter in Physics and Astrophysics, edited by J. Madsen and P. Haensel (Nucl. Phys. B (Proc. Suppl.), 24B, 1991), pp. 40-44.

[71] C. Kettner, F. Weber, M. K. Weigel, and N. K. Glendenning, Phys. Rev. D 51, 1440 (1995).

[72] N. K. Glendenning, C. Kettner, and F. Weber, Phys. Rev. Lett. 74, 3519 (1995).

[73] N. K. Glendenning, C. Kettner, and F. Weber, Astrophys. J. 450, 253 (1995).

[74] O. G. Benvenuto and J. E. Horvath, Mon. Not. R. Ast. Soc. 247, 584 (1990).

[75] F. C. Michel, Phys. Rev. Lett. 60, 677 (1988). 
[76] N. K. Glendenning, Phys. Rev. D 46, 1274 (1992).

[77] H. Heiselberg, C. J. Pethick, and E. F. Staubo, Phys. Rev. Lett. 70, 1355 (1993).

[78] N. K. Glendenning and S. Pei, Phys. Rev. C 52, 2250 (1995).

[79] M. B. Christiansen and N. K. Glendenning, Phys. Rev. C 56, 2858 (1997).

[80] J. M. Lattimer, C. J. Pethick, M. Prakash, and P. Haensel, Phys. Rev. Lett. 66, 2701 (1991).

[81] C. J. Pethick, Rev. Mod. Phys. 64, 1133 (1992).

[82] C. Schaab, B. Hermann, F. Weber, and M. K. Weigel, Astrophys. J. 480, L111 (1997).

[83] Y. F. Huang and T. Lu, Astron. Astrophys. 325, 189 (1997).

[84] T. Lu, preprint astro-ph/9807052.

[85] M. A. Alpar, Phys. Rev. Lett. 58, 2152 (1987).

[86] N. K. Glendenning and F. Weber, Astrophys. J. 400, 647 (1992).

[87] B. Datta, P. K. Sahu, J. D. Anand, and A. Goyal, Phys. Lett. B 283, 313 (1992).

[88] Q. D. Wang and T. Lu, Phys. Lett. B 148, 211 (1984).

[89] R. F. Sawyer, Phys. Lett. B 233, 412 (1989).

[90] C. Cutler, L. Lindblom, and R. J. Splinter, Astrophys. J. 363, 603 (1990).

[91] J. Madsen, Phys. Rev. D 46, 3290 (1992).

[92] J. L. Zdunik, in Strange Quark Matter in Physics and Astrophysics, edited by J. Madsen and P. Haensel (Nucl. Phys. B (Proc. Suppl.), 24B, 1991), pp. $119-124$.

[93] M. Colpi and J. C. Miller, Astrophys. J. 388, 513 (1992).

[94] N. Andersson, Astrophys. J. in press (1998). Preprint gr-qc/9706075.

[95] J. L. Friedman and S. M. Morsink, Astrophys. J. in press (1998). Preprint gr-qc/9706073.

[96] L. Lindblom, B. J. Owen, and S. M. Morsink, Phys. Rev. Lett. 80, 4843 (1998). 
[97] J. Madsen, Phys. Rev. Lett. submitted (1998). Preprint astro-ph/9806032.

[98] H. Heiselberg and C. J. Pethick, Phys. Rev. D 48, 2916 (1993).

[99] P. Haensel and A. J. Jerzak, Acta Phys. Pol. B 20, 141 (1989).

[100] C. Alcock, E. Farhi, and A. Olinto, Phys. Rev. Lett. 57, 2088 (1986).

[101] J. E. Horvath, H. Vucetich, and O. G. Benvenuto, Mon. Not. R. Ast. Soc. 262, 506 (1993).

[102] K. S. Cheng and Z. G. Dai, Phys. Rev. Lett. 80, 18 (1998).

[103] K. S. Cheng, Z. G. Dai, D. M. Wei, and T. Lu, Science 280, 407 (1998).

[104] P. Haensel, B. Paczyński, and P. Amsterdamski, Astrophys. J. 375, 209 (1991).

[105] X.-D. Li, Z.-G. Dai, and Z.-R. Wang, Astron. Astrophys. 303, L1 (1995).

[106] J. Madsen, Astron. Astrophys. 318, 466 (1997).

[107] G. Baym et al., Phys. Lett. B 160, 181 (1985).

[108] A. V. Olinto, Phys. Lett. B 192, 71 (1987).

[109] H. Heiselberg, G. Baym, and C. J. Pethick, in Strange Quark Matter in Physics and Astrophysics, edited by J. Madsen and P. Haensel (Nucl. Phys. B (Proc. Suppl.), 24B, 1991), pp. 144-147.

[110] M. L. Olesen and J. Madsen, in Strange Quark Matter in Physics and Astrophysics, edited by J. Madsen and P. Haensel (Nucl. Phys. B (Proc. Suppl.), 24B, 1991), pp. 170-174.

[111] A. Olinto, in Strange Quark Matter in Physics and Astrophysics, edited by J. Madsen and P. Haensel (Nucl. Phys. B (Proc. Suppl.), 24B, 1991), pp. 103-109.

[112] J. E. Horvath and O. G. Benvenuto, Phys. Lett. B 213, 516 (1988).

[113] M. L. Olesen and J. Madsen, Phys. Rev. D 49, 2698 (1994).

[114] M. I. Krivoruchenko and B. V. Martemyanov, Astrophys. J. 378, 628 (1991).

[115] J. E. Horvath, O. G. Benvenuto, and H. Vucetich, Phys. Rev. D 45, 3865 (1992).

[116] K. Iida and K. Sato, Prog. Theor. Phys. 98, 277 (1997).

[117] K. Iida, Prog. Theor. Phys. 98, 739 (1997). 
[118] A. De Rújula and S. L. Glashow, Nature 312, 734 (1984).

[119] P. B. Price, Phys. Rev. D 38, 3813 (1988).

[120] D. M. Lowder, in Strange Quark Matter in Physics and Astrophysics, edited by J. Madsen and P. Haensel (Nucl. Phys. B (Proc. Suppl.), 24B, 1991), pp. 177-183.

[121] J. Madsen, Phys. Rev. Lett. 61, 2909 (1988).

[122] M. Jändel, Z. Phys. C 40, 599 (1988).

[123] R. R. Caldwell and J. L. Friedman, Phys. Lett. 264B, 143 (1991).

[124] R. N. Boyd and T. Saito, Phys. Lett. B 298, 6 (1993).

[125] J. D. Bjorken and L. D. McLerran, Phys. Rev. D 20, 2353 (1979).

[126] F. Halzen and H. C. Liu, Phys. Rev. D 32, 1716 (1985).

[127] M. S. Desai and G. L. Shaw, in Strange Quark Matter in Physics and Astrophysics, edited by J. Madsen and P. Haensel (Nucl. Phys. B (Proc. Suppl.), 24B, 1991), pp. 207-210. 